\title{
EXISTENCE AND UNIQUENESS THEOREMS FOR EXTENSIONS OF ZERO-DIMENSIONAL COMPACT METRIC SPACES
}

BY

\author{
R. S. PIERCE
}

1. Introduction. One of the central problems in the theory of Boolean algebras is the classification of all countable Boolean algebras. Using the Stone representation theorem, this objective can be translated into topological form: classify the homeomorphism types of all zero-dimensional, compact metric spaces. The purpose of this paper is to prove an existence theorem and a uniqueness theorem which shed some light on this classification problem.

For any metric space $X$, and for any ordinal number $\xi$, let $X^{(\xi)}$ denote the $\xi$ th topological derivative of $X$. The definition of this concept will be recalled in detail below. The space $X$ is called perfect if $X^{(1)}=X$. The opposite extreme occurs when $X^{(\xi)}=\varnothing$ for some $\xi$. In this case, $X$ is said to be scattered. Any nonempty, perfect, zero-dimensional compact metric space is homeomorphic to the Cantor discontinuum $\mathscr{D}$. A scattered, zero-dimensional compact metric space is homeomorphic to the ordered space $\omega^{\mu} \cdot n+1$ for some uniquely determined, countable ordinal number $\mu$, and some unique natural number $n$. Thus, the classification problem for zero-dimensional, compact metric spaces has a satisfactory solution for either perfect or scattered spaces. The spaces between these extremes have a unique decomposition as a disjoint union of a nonempty, closed, perfect subspace, and a nonempty, open, scattered subspace. The classification of these subspaces can be handled by the results for the perfect and scattered cases, so that the problem is one of determining how the Cantor discontinuum can be extended by adjoining a particular scattered space. It is to this question that our main theorems are addressed.

THEOREM 1.1. Let $X$ and $Y$ be zero-dimensional, compact metric spaces. Let $Z \subseteq X$ and $W \subseteq Y$ be closed subsets such that $X-Z$ and $Y-W$ are scattered. Suppose that $\varphi: Z \rightarrow W$ is a homeomorphism. Then $\varphi$ can be extended to a homeomorphism of $X$ onto $Y$ if and only if

(a) $X-Z \simeq Y-W$, and

(b) $\varphi\left(Z \cap\left(X^{(\xi)}-Z\right)^{-}\right)=W \cap\left(Y^{(\xi)}-W\right)^{-}$for all ordinal numbers $\xi$.

The conditions (a) and (b) of Theorem 1.1 are plainly necessary; the essential content of the theorem is the fact that they are also sufficient. 
THEOREM 1.2. Let $Z$ be a zero-dimensional, compact metric space, and suppose that $Z \supseteq Z_{0} \supseteq Z_{1} \supseteq \cdots \supseteq Z_{\xi} \supseteq \cdots, \xi<\mu$, where each $Z_{\xi}$ is closed and nonempty. Then there is a zero-dimensional compact metric space $X \supseteq Z$ such that

(a) $X-Z \simeq \omega^{\mu}$, and

(b) $Z \cap\left(X^{(\xi)}-Z\right)^{-}=Z_{\xi}$ for all $\xi<\mu$.

This result clearly shows the complexity of the class of zero-dimensional, compact metric spaces.

2. Terminology and background. We will use the term Boolean space to designate a zero-dimensional, compact Hausdorff space. The name Stone space will mean a locally compact, zero-dimensional, Hausdorff space. The class of all metrizable Boolean spaces will be denoted by $\mathfrak{S}$. By the Urysohn metrization theorem, a Boolean space $X$ belongs to $\subseteq$ if and only if $X$ satisfies the second axiom of countability.

It is well known that every $X \in \cong$ is homeomorphic to a closed subset of the Cantor discontinuum $\mathscr{D}$. This fact has the useful consequence that if $X \in \mathfrak{S}$, then there is a metric $d$ for $X$ that satisfies: $d(x, y)=d(x, z)$ implies $y=z$, for all $x, y$, and $z$ in $X$. Indeed, $\mathscr{D}$ has such a metric, and therefore so does every closed subset of $\mathscr{D}$. (See [1] for the proof of this fact.) A metric with this property will be called a $U . D$. metric (U.D. standing for unique distance). Any U.D. metric on $X$ determines retractions of $X$ onto each of its nonempty, closed subspaces $Y$. Specifically, let $\pi$ be the mapping that sends each $x \in X$ to the unique point of $Y$ which lies closest to $x$. We will refer to $\pi$ as the "nearest point retraction of $X$ onto $Y$." Note that by the definition of $\pi, d(x, \pi(x))=d(x, Y)$, for all $x \in X$.

The transfinite sequence of topological derivatives are defined recursively for an arbitrary space $X$ as follows. For any subset $Y$ of $X$, let $Y^{\prime}$ denote the set of all accumulation points of $Y$, that is, $Y^{\prime}$ is the complement in $Y$ of the set of points which are isolated in the relative topology of $Y$. Then we define

$X^{(0)}=X$,

$X^{(\xi+1)}=\left(X^{(\xi)}\right)^{\prime}$,

$X^{(\eta)}=\bigcap_{\xi<\eta} X^{(\xi)}$, when $\eta$ is a limit ordinal.

Several elementary observations follow from this definition:

$X^{(\xi)}$ is closed in $X$;

$\xi \leqq \eta$ implies $X^{(\xi)} \supseteq X^{(\eta)}$;

$X^{(\xi)}-X^{(\xi+1)}$ is the set of isolated points of $X^{(\xi)}$ (with its relative topology);

$\left(X^{(\xi)}\right)^{(\eta)}=X^{(\xi+\eta)}$;

$Y \subseteq Z$ implies $Y^{(\xi)} \subseteq Z^{(\xi)}$.

These facts will be used without citation. Slightly deeper properties are listed in the following proposition.

Lemma 2.1. Assume that $X$ is a Hausdorff space.

(a) There is a smallest ordinal number $\lambda$ such that $X^{(\lambda)}=X^{(\lambda+1)}$; if $\xi \geqq \lambda$, then $X^{(\xi)}=X^{(\lambda)}$. 
(b) If $X$ is compact, and $X^{(\xi)}=\varnothing$ for some $\xi$, then there is a nonlimit ordinal $\lambda$ such that $X^{(\lambda)}=\varnothing$ and $X^{(\lambda-1)}$ is finite and not empty.

(c) If $Y$ is open in $X$, then $Y^{(\xi)}=Y \cap X^{(\xi)}$ for all $\xi$.

(d) If $X=\bigcup_{i \in I} Y_{i}$, where each $Y_{i}$ is open in $X$, and $Y_{i} \cap Y_{j}=\varnothing$ for $i \neq j$, then $X^{(\xi)}=\bigcup_{i \in I} Y_{i}^{(\xi)}$.

(e) If $\varphi: X \rightarrow Y$ is a homeomorphism, then $\varphi\left(X^{(\xi)}\right)=Y^{(\xi)}$.

The proofs of these facts can be found in [2] and [3].

As is customary, each ordinal number will be identified with the set of all smaller ordinals. Consequently, ordinal numbers are themselves well ordered sets. This convention can cause some confusion. For example in a discussion of an ordinal number $\xi$, it is sometimes necessary to decide from context whether $\xi$ is to be considered as the label for an ordered set, or rather as an object of another set. Usually, this decision can be made without difficulty.

If $V$ is a totally ordered set, and if $u<v$ are elements of $V$, then the various intervals determined by $u$ and $v$ are denoted in the usual way:

$$
\begin{array}{ll}
{[u, v]=\{x \in V \mid u \leqq x \leqq v\},} & {[u, v)=\{x \in V \mid u \leqq x<v\}} \\
(u, v]=\{x \in V \mid u<x \leqq v\}, & \text { and } \quad(u, v)=\{x \in V \mid u<x<v\} .
\end{array}
$$

Summary of notation.

(a) $\mathfrak{S}=$ class of all metrizeable Boolean spaces (i.e., zero-dimensional, compact metric spaces).

(b) $\simeq$ represents homeomorphism of topological spaces.

(c) $\sim$ represents order isomorphism of ordered sets.

(d) If $Y$ is a subset of the topological space $X$, the closure of $Y$ in $X$ is denoted by $Y^{-}$.

(e) If $Y$ is a subset of a space with a metric $d$, then the diameter of $Y$ is denoted by $\operatorname{diam}(Y)$. That is, $\operatorname{diam}(Y)=\sup \{d(x, y) \mid x, y \in Y\}$.

(f) Let $\varphi_{i}: X_{i} \rightarrow Y, i \in I$, be a set of mappings with disjoint domains $\left(X_{i} \cap X_{j}=\varnothing\right.$ for $i \neq j)$. Define $\bigcup_{i \in I} \varphi_{i}: \bigcup_{i \in I} X_{i} \rightarrow Y$ by $\left(\bigcup_{i \in I} \varphi_{i}\right)(x)=\varphi_{j}(x)$ for all $x \in X_{j}$. For the case of two mappings $\varphi$ and $\psi$ with disjoint domains, this combined mapping will be written $\varphi \cup \psi$.

3. Scattered Stone spaces. For the proof of Theorem 1.1 it is necessary to know something about the structure of scattered Stone spaces with a countable basis. The result that is needed will be presented in this section.

Definition 3.1. Let $X$ be a Stone space. Define

$\lambda(X)=\min \left\{\xi \mid X^{(\xi+1)}=X^{(\xi)}\right\}$,

$\mu(X)=\min \left\{\xi \mid X^{(\xi)}-X^{(\lambda(X))}\right.$ is compact $\}$.

Note that $\mu(X)$ is well defined and $\leqq \lambda(X)$. Moreover, the space $X$ is scattered exactly in the case that $X^{(\lambda(X))}=\varnothing$, and indeed $\lambda(X)$ is the first vanishing derivative of $X$. 
Proposition 3.2. Let $X$ be a scattered Stone space with a countable basis of open sets. Then there is a countable ordinal number $\alpha$ and a natural number $n$ such that $X$ is homeomorphic to $\left(\omega^{\alpha} \cdot n+1\right)-\left\{\omega^{\mu(X)}\right\}$ with the relative topology inherited from the order topology of $\omega^{\alpha} \cdot n+1$. Moreover, either

(a) $\lambda(X)=\mu(X)=\alpha$ and $n=1$, or

(b) $\lambda(X)=\alpha+1, \mu(X)=\alpha$, and $n=\left|X^{(\alpha)}\right|+1$, or

(c) $\lambda(X)=\alpha+1, \mu(X)<\alpha$, and $n=\left|X^{(\alpha)}\right|$.

Proof. Let $Y$ be the one point compactification of $X$, say $Y=X \cup\{\infty\}$. Then $Y \in \subseteq$, and $Y^{(\lambda(X)+1)}=\varnothing$ by Lemma 2.1(c) and the fact that $X$ is scattered and open in $Y$. Consequently, there is a countable ordinal $\alpha$, a natural number $n$, and a homeomorphism $\theta: Y \rightarrow \omega^{\alpha} \cdot n+1$ (see [3] or [4]). Let $\theta(\infty)=\xi$, where $\xi \leqq \omega^{\alpha} \cdot n$. Without loss of generality, it can be assumed that $\xi=\omega^{\beta}$ for some ordinal number $\beta \leqq \alpha$. In fact, any $\xi$ can be written in the form $\eta+\omega^{\beta}$, and it is easy to see that there is a homeomorphism of $\omega^{\alpha} \cdot n+1$ onto itself which carries $\eta+\omega^{\beta}$ to $\omega^{\beta}$. By Lemma 2.1(c) and (e), $\theta\left(X^{(\xi)}\right)=\left(\omega^{\alpha} \cdot n+1\right)^{(\xi)}-\left\{\omega^{\beta}\right\}$ for all $\xi$. The results in $\S 4$ of [3] shows that

(i) $\omega^{\beta} \in\left(\omega^{\alpha} \cdot n+1\right)^{(\xi)}$ if and only if $\xi \leqq \beta$, and

(ii) $\left(\omega^{\alpha} \cdot n+1\right)^{(\alpha)}=\left\{\omega^{\alpha} \cdot 1, \omega^{\alpha} \cdot 2, \omega^{\alpha} \cdot 3, \ldots, \omega^{\alpha} \cdot n\right\}$.

From (i) it is clear that $X^{(\xi)}-X^{(\lambda(X))}=X^{(\xi)}$ is compact if and only if $\xi \geqq \beta$. Hence, $\beta=\mu(X)$. Using this observation and (ii), the alternatives (a), (b), or (c) are easily obtained.

It is evident that the natural number $n=n(X)$ defined in Proposition 3.2 is an invariant of the space $X$, and that the triple $(\lambda(X), \mu(X), n(X))$ completely determines $X$. Thus, we obtain a complete system of invariants for scattered Stone spaces with countable bases of open sets.

Now consider the situation described in Theorem 1.1, that is, let $X \in \mathfrak{S}$, and suppose that $Z$ is a closed subset of $X$ such that $X-Z$ is scattered. Then $X-Z$ is a scattered Stone space with a countable basis. By Proposition 3.2, there is a homeomorphism $\theta:(X-Z) \rightarrow\left(\omega^{\alpha} \cdot n+1\right)-\left\{\omega^{\mu}\right\}$, where $\mu=\mu(X-Z)$, and either

(a) $\mu=\alpha=\lambda(X-Z)$ and $n=1$, or

(b) $\lambda(X-Z)=\alpha+1, \mu=\alpha$, and $n=\left|(X-Z)^{(\alpha)}\right|+1$, or

(c) $\lambda(X-Z)=\alpha+1, \mu<\alpha$, and $n=\left|(X-Z)^{(\alpha)}\right|$.

Let $U=\theta^{-1}\left(\left\{\xi \mid \omega^{\mu}<\xi \leqq \omega^{\alpha} \cdot n\right\}\right)$. Then $U$ is a compact open subset of $X$, and in the respective cases (a), (b) and (c) we have

$$
U=\varnothing, \quad U \simeq \omega^{\alpha} \cdot(n-1)+1, \quad \text { and } \quad U \simeq \omega^{\alpha} \cdot n+1 .
$$

It is clear that $Z \subseteq X-U$, and $Z \cap\left((X-U)^{(\xi)}-Z\right)^{-}=Z \cap\left(X^{(\xi)}-Z\right)^{-}$for all $\xi$. From this observation, it follows that for the proof of Theorem 1.1 we may assume without loss of generality that $X-Z$ and $Y-W$ are both homeomorphic to the ordered set consisting of all ordinal numbers less than $\omega^{\mu}$.

4. Proof of 1.2. Theorems 1.1 and 1.2 are both proved by transfinite induction on $\mu$. We begin with 1.2, whose proof is somewhat easier than the proof of 1.1. 
It is convenient to get a different approach to the problem. Our first lemma accomplishes this.

Lemma 4.1. Let $X \in \mathfrak{S}$, and suppose that $Z \supseteq Z_{0} \supseteq Z_{1} \supseteq \cdots \supseteq Z_{\xi} \supseteq \cdots, \xi<\mu$, where each $Z_{\xi}$ is closed and nonempty. Assume that there exists a continuous mapping $\varphi: \omega^{\mu} \rightarrow Z$ satisfying

(a) $\varphi\left(\left(\omega^{\mu}\right)^{(\xi)}\right) \subseteq Z_{\xi}$ for all $\xi<\mu$, and

(b) for all $\xi<\mu$, if $y \in Z_{\xi}$, then there exists $\left\{\eta_{i} \mid i<\omega\right\} \subseteq\left(\omega^{\mu}\right)^{(\xi)}$ such that $\lim _{i \rightarrow \omega} \eta_{i}$ $=\omega^{\mu}$ and $\lim _{i \rightarrow \omega} \varphi\left(\eta_{i}\right)=y$.

Then there exists $X \in \subseteq$ satisfying $X \supseteq Z, X-Z \simeq \omega^{\mu}$, and $Z \cap\left(X^{(\xi)}-Z\right)^{-}=Z_{\xi}$ for all $\xi<\mu$.

Proof. Define $X$ to be the following subspace of $\left(\omega^{\mu}+1\right) \times Z$ :

$$
X=\left\{(\eta, \varphi(\eta)) \mid \eta<\omega^{\mu}\right\} \cup\left(\left\{\omega^{\mu}\right\} \times Z\right) .
$$

Using the continuity of $\varphi$, it is easy to show that $X$ is closed in $\left(\omega^{\mu}+1\right) \times Z$. Hence, $X \in \mathfrak{S}$. Plainly, we can identify each $y \in Z$ with $\left(\omega^{\mu}, y\right) \in X$, so that $Z$ is embedded in $X$. The continuity of $\varphi$ implies that the inverse of the continuous map $(\eta, \varphi(\eta))$ $\rightarrow \eta\left(\eta<\omega^{\mu}\right)$ is continuous. Thus, the open set $U=\left\{(\eta, \varphi(\eta)) \mid \eta<\omega^{\mu}\right\}$ is homeomorphic to $\omega^{\mu}$, and by Lemma $2.1(\mathrm{c})$,

$$
X^{(\xi)}-Z=U \cap X^{(\xi)}=U^{(\xi)}=\left\{(\eta, \varphi(\eta)) \mid \eta \in\left(\omega^{\mu}\right)^{(\xi)}\right\} .
$$

Therefore, by (a), $\left(X^{(\xi)}-Z\right)^{-} \subseteq\left(\omega^{\mu}+1\right) \times Z_{\xi}$ and $Z \cap\left(X^{(\xi)}-Z\right)^{-} \subseteq Z_{\xi}$.

On the other hand, if $y \in Z_{\xi}$, then by (b) there is a sequence $\left\{\eta_{i} \mid i<\omega\right\} \subseteq\left(\omega^{\mu}\right)^{(\xi)}$ such that $\lim _{i \rightarrow \omega} \eta_{i}=\omega^{\mu}$ and $\lim _{i \rightarrow \omega} \varphi\left(\eta_{i}\right)=y$. Therefore $\left\{\left(\eta_{i}, \varphi\left(\eta_{i}\right)\right) \mid i<\omega\right\}$ is a sequence in $X^{(\xi)}-Z$ which satisfies $\lim _{i \rightarrow \omega}\left(\eta_{i}, \varphi\left(\eta_{i}\right)\right)=\left(\omega^{\mu}, y\right)$. This shows that $Z \cap\left(X^{(\xi)}-Z\right)^{-}=Z_{\xi}$ for all $\xi<\mu$.

Our task is now reduced to the job of constructing a continuous mapping $\varphi: \omega^{\mu} \rightarrow Z$ which satisfies 4.1(a) and 4.1(b).

It is helpful to introduce some notation. Let $V$ be an ordered set, and $\left\{v_{i} \mid i<\omega\right\}$ $\subseteq V$. We will write $\lim _{i \rightarrow \omega} v_{i}=\infty$ as an abbreviation for the sentence "for all $v \in V$, there exists $n<\omega$ such that $i>n$ implies $v_{i} \geqq v$."

Let $V$ be an ordered set (topologized with the order topology), $Z$ a topological space, and $\varphi: V \rightarrow Z$ a continuous mapping. For each ordinal number $\xi$, define

$$
\Phi_{\xi}(\varphi)=\bigcap_{v \in V}\left(\varphi\left(V^{(\xi)} \cap\{w \in V \mid w \geqq v\}\right)\right)^{-} .
$$

The case which interests us occurs when $V$ is countable and $Z$ is a Boolean space with a countable basis. For this situation, $\Phi_{\xi}(\varphi)$ can be characterized as the set of all $y \in Z$ such that there exists a sequence $\left\{v_{i} \mid i<\omega\right\} \subseteq V^{(\xi)}$ satisfying $\lim _{i \rightarrow \omega} v_{i}=\infty$, and $\lim _{i \rightarrow \omega} \varphi\left(v_{i}\right)=y$. In particular, the condition (b) of Lemma 4.1 can be reformulated as: $\Phi_{\xi}(\varphi)=Z_{\xi}$ for all $\xi<\mu$.

LEMMA 4.2. Let $V$ be an ordered set, $V=\bigcup_{i \in I} V_{i}$, where each $V_{i}$ is open in $V$, $V_{i} \cap V_{j}=\varnothing$ for $i \neq j$, and each $V_{i}$ is cofinal in $V$. Suppose that for each $i \in I, \varphi_{i}$ is a 
continuous mapping of $V_{i}$ to the space $Z$. Define $\varphi=\bigcup_{i \in I} \varphi_{i}$. Then $\varphi$ is a continuous mapping of $V$ to $Z$, and $\Phi_{\xi}(\varphi) \supseteq \bigcup_{i \in I} \Phi_{\xi}\left(\varphi_{i}\right)$ for all $\xi$. If $I$ is finite, then $\Phi_{\xi}(\varphi)$ $=\bigcup_{i \in I} \Phi_{\xi}\left(\varphi_{i}\right)$.

Proof. If $N$ is open in $Y$, then $\varphi^{-1}(N)=\bigcup_{i \in I} \varphi_{i}^{-1}(N)$ is open in $V$ (since each $V_{i}$ is open, and each $\varphi_{i}$ is continuous). Thus, $\varphi$ is continuous. Using the fact that $V_{i}$ is confinal in $V$, we obtain

$$
\begin{aligned}
\Phi_{\xi}\left(\varphi_{i}\right) & =\bigcap_{v \in V_{i}}\left(\varphi_{i}\left(V_{i}^{(\xi)} \cap\left\{w \in V_{i} \mid w \geqq v\right\}\right)\right)^{-} \\
& =\bigcap_{v \in V}\left(\varphi_{i}\left(V_{i}^{(\xi)} \cap\left\{v \in V_{i} \mid w \geqq v\right\}\right)\right)^{-} \subseteq \bigcap_{v \in V}\left(\varphi\left(V^{(\xi)} \cap\{w \in V \mid w \geqq v\}\right)\right)^{-} \\
& =\Phi_{\xi}(\varphi) .
\end{aligned}
$$

Since $i \in I$ was arbitrary, we have $\Phi_{\xi}(\varphi) \supseteq \bigcup_{i \in I} \Phi_{\xi}\left(\varphi_{i}\right)$. For the proof of the last statement, it can be assumed that $I=\{0,1\}$. In this case,

$$
\begin{aligned}
\Phi_{\xi}(\varphi) & =\bigcap_{v \in V}\left(V^{(\xi)} \cap\{w \in V \mid w \geqq v\}\right)^{-} \\
& =\bigcap_{v \in V}\left(\left(\varphi_{0}\left(V_{0}^{(\xi)} \cap\{w \in V \mid w \geqq v\}\right)\right)^{-} \cup\left(\varphi_{1}\left(V_{1}^{(\xi)} \cap\{w \in V \mid w \geqq v\}\right)\right)^{-}\right),
\end{aligned}
$$

since $V^{(\xi)}=V_{0}^{(\xi)} \cup V_{1}^{(\xi)}$ by 2.1. Using the fact that if $\left\{A_{v} \mid v \in V\right\}$ and $\left\{B_{v} \mid v \in V\right\}$ are nested families of sets (i.e. $v<v^{\prime}$ implies $A_{v} \supseteq A_{v^{\prime}}$ and $\left.B_{v} \supseteq B_{v^{\prime}}\right)$, then $\bigcap_{v \in V}\left(A_{v} \cup B_{v}\right)$ $=\left(\bigcap_{v \in V} A_{v}\right) \cup\left(\bigcap_{v \in V} B_{v}\right)$, we obtain the desired result:

$$
\begin{aligned}
\Phi_{\xi}(\varphi) & =\left(\bigcap_{v \in V}\left(\varphi_{0}\left(V_{0}^{(\xi)} \cap\{w \in V \mid w \geqq v\}\right)\right)^{-}\right) \cup\left(\bigcap_{v \in V}\left(\left(V_{1}^{(\xi)} \cap\{w \in V \mid w \geqq v\}\right)\right)^{-}\right) \\
& =\Phi_{\xi}\left(\varphi_{0}\right) \cup \Phi_{\xi}\left(\varphi_{1}\right)
\end{aligned}
$$

(because $V_{0}$ and $V_{1}$ are cofinal in $V$ ).

Lemma 4.3. Let $\mu$ be a countable ordinal number, and let $0 \leqq \beta<\alpha<\mu$. Then there exist subsets $V_{0}, V_{1}$ of $\omega^{\mu}$ such that

(a) $V_{0} \cap V_{1}=\varnothing, V_{0} \cup V_{1}=\omega^{\mu}$,

(b) $V_{0}$ and $V_{1}$ are open and cofinal in $\omega^{\mu}$,

(c) $V_{0} \sim \omega^{\alpha}, V_{1} \sim \omega^{\mu}$, and

(d) $V_{1} \subseteq\left[\omega^{\beta}, \omega^{\mu}\right)$.

Proof. Let $\left\{\gamma_{i} \mid i<\omega\right\}$ be a strictly increasing sequence of ordinal numbers satisfying $\gamma_{0} \geqq \omega^{\beta}$ and $\lim _{i \rightarrow \omega} \gamma_{i}=\omega^{\alpha}$. Let $\left\{\delta_{i} \mid 1 \leqq i<\omega\right\}$ be a strictly increasing sequence of ordinal numbers such that $\delta_{1} \geqq \omega^{\alpha}$ and $\lim _{i \rightarrow \omega} \delta_{i}=\omega^{\mu}$. Define

$$
V_{0}=\left[0, \gamma_{0}\right] \cup \bigcup_{1 \leqq i<\omega}\left(\delta_{i}, \delta_{i}+\gamma_{i}\right], \quad V_{1}=\left(\gamma_{0}, \delta_{1}\right] \cup \bigcup_{1 \leqq i<\omega}\left(\delta_{i}+\gamma_{i}, \delta_{i+1}\right] .
$$

Plainly, (a), (b), and (d) are satisfied, and (c) is easily obtained from the identities $\sum_{i<\omega} \gamma_{i}=\omega^{\alpha}$ and $\sum_{1 \leqq i<\omega} \delta_{i}=\omega^{\mu}$.

LeMma 4.4. Let $\mu$ be a countable limit ordinal, and let $\left\{\alpha_{i} \mid i<\omega\right\}$ be a strictly increasing sequence of ordinal numbers such that $\alpha_{0}>0$ and $\lim _{i \rightarrow \omega} \alpha_{i}=\mu$. Then there 
is a family $\left\{V_{i} \mid i<\omega\right\}$ of subsets of $\omega^{\mu}$ such that

(a) $V_{i} \cap V_{j}=\varnothing$ if $i \neq j$, and $\bigcup_{i<\omega} V_{i}=\omega^{\mu}$,

(b) all $V_{i}$ are open and cofinal in $\omega^{\mu}$, and

(c) $V_{i} \sim \omega^{\alpha_{i}}$ for all $i$.

Proof. Applying 4.3 with $\beta=0, \alpha=\alpha_{0}$ and $\mu$, we can obtain sets $V_{0}$ and $U_{0}$ which are open and cofinal in $\omega^{\mu}$, and satisfy $V_{0} \cap U_{0}=\varnothing, V_{0} \cup U_{0}=\omega^{\mu}, V_{0} \sim \omega^{\alpha}$, and $U_{0} \sim \omega^{\mu}$. Reapply 4.3 to the set $U_{0}$ with $\beta=\alpha_{0}, \alpha=\alpha_{1}$, and $\mu$. We obtain sets $V_{1}$ and $U_{1}$ which are open and cofinal in $U_{0}$ (consequently, also open and cofinal in $\left.\omega^{\mu}\right)$, and satisfy $V_{1} \cap U_{1}=\varnothing, V_{1} \cup U_{1}=U_{0}, V_{1} \sim \omega^{\alpha}, U_{1} \sim \omega^{\mu}$, and $U_{1}$ does not meet the initial segment $W_{0}$ of $U_{0}$, such that $W_{0} \sim \omega^{\alpha}$. This last condition implies that $U_{1} \subseteq\left[\omega^{\alpha_{0}}, \omega^{\mu}\right.$ ) (see, for example, [5, Lemma 3.4, p. 8, and Proposition 3.2, p. 7]). Continue this process. After $n+1$ steps we have sets $V_{0}, V_{1}, \ldots, V_{n}, U_{n}$ which are open and cofinal in $\omega^{\mu}$, and satisfy $V_{i} \cap V_{j}=\varnothing$ for $i \neq j, V_{i} \cap U_{n}=\varnothing$ for all $i, V_{0} \cup V_{1} \cup \cdots \cup V_{n} \cup U_{n}=\omega^{\mu}, V_{i} \sim \omega^{\alpha_{i}}$ for all $i, U_{n} \sim \omega^{\mu}$, and $U_{n} \subseteq$ $\left\{\eta<\omega^{\mu} \mid \eta \geqq \omega^{\alpha_{n-1}}\right\}$. Since $\lim _{i \rightarrow \omega} \omega^{\alpha_{i}}=\omega^{\mu}$, it is clear that if the construction is repeated indefinitely, the collection $\left\{V_{i} \mid i<\omega\right\}$ will satisfy the conditions (a), (b), and (c).

We now proceed with the proof of 1.2. Let $Z$ and $Z_{\xi}, \xi<\mu$, be given as in the statement of the theorem. It will be shown by induction on $\mu$ that there exists a continuous mapping $\varphi: \omega^{\mu} \rightarrow Z$ which satisfies the conditions 4.1(a) and 4.1(b). If $\mu=0$, there is nothing to prove.

Case: $\mu=1$. Let $\left\{y_{i} \mid i<\omega\right\}$ be a dense subset of $Z_{0}$. Let $\rho: \omega \rightarrow \omega \times \omega$ be one-toone and onto, and let $\pi: \omega \times \omega \rightarrow \omega$ be the projection $\pi(r, s)=r$. Define $\varphi(n)$ $=y_{\pi \rho(n)}$. Note that for any $i<\omega,(\pi \rho)^{-1}(\{i\})$ is an infinite subset of $\omega$. From this observation and the denseness of $\left\{y_{i} \mid i<\omega\right\}$, it follows that $\varphi$ satisfies 4.1(b). Clearly, 4.1(a) is satisfied. Finally, $\varphi$ is automatically continuous, since $\omega$ is discrete in its order topology.

Induction step: $\mu=\alpha+1$. By 4.3 there exist subsets $V_{0}, V_{1}$ which are open and cofinal in $\omega^{\mu}$, and satisfy $V_{0} \cap V_{1}=\varnothing, V_{0} \cup V_{1}=\omega^{\mu}, V_{0} \sim \omega^{\alpha}$, and $V_{1} \sim \omega^{\mu}$. By the induction hypothesis, there exists a continuous mapping $\varphi_{0}: V_{0} \rightarrow Z$ such that $\varphi_{0}\left(V_{0}^{(\xi)}\right) \subseteq Z_{\xi}$ and $\Phi_{\xi}\left(\varphi_{0}\right)=Z_{\xi}$ for $\xi<\alpha$. Since $V_{0} \sim \omega^{\alpha}$, it is clear that $V_{0}^{(\alpha)}=\varnothing$. Since $V_{1} \sim \omega^{\mu}$, there is a one-to-one, order preserving mapping $\theta$ of $V_{1}$ onto $\omega^{\mu}=\omega^{\alpha+1}$. By the case $\mu=1$, there is a continuous mapping $\psi: \omega \rightarrow Z$ such that $\psi(\omega) \subseteq Z_{\alpha}$ and $\Phi_{0}(\psi)=Z_{\alpha}$. Define $\chi: \omega^{\mu} \rightarrow Z$ by $\chi\left(\omega^{\alpha} \cdot n\right)=\psi(n)$ for $n<\omega$, and $\chi\left(\omega^{\alpha} \cdot n+\gamma\right)$ $=\psi(n+1)$ for $n<\omega$ and $0<\gamma<\omega^{\alpha}$. Since $\chi$ is constant on each of the closed intervals $\left(\omega^{\alpha} \cdot n, \omega^{\alpha} \cdot(n+1)\right]$, this mapping is continuous. Let $\varphi_{1}=\chi \theta: V_{1} \rightarrow Z$. Plainly, $\varphi_{1}\left(\left(\omega^{\mu}\right)^{(\xi)}\right) \subseteq Z_{\alpha}$ for all $\xi<\mu$, and it is easy to see that $\Phi_{\xi}\left(\varphi_{1}\right)=Z_{\alpha}$ for all $\alpha<\mu$. Since $\theta$ and $\chi$ are continuous, so is $\varphi_{1}$. Finally, let $\varphi=\varphi_{0} \cup \varphi_{1}$. Using Lemma 2.1, we have $\varphi\left(\left(\omega^{\mu}\right)^{(\xi)}\right)=\varphi\left(V_{0}^{(\xi)} \cup V_{1}^{(\xi)}\right)=\varphi_{0}\left(V_{0}^{(\xi)}\right) \cup \varphi_{1}\left(V_{1}^{(\xi)}\right) \subseteq Z_{\alpha} \cup Z_{\xi}=Z_{\xi}$ for all $\xi \leqq \alpha$ (that is, all $\xi<\mu$ ). In particular, $\Phi_{\xi}(\varphi) \subseteq Z_{\xi}$ for all $\xi<\mu$, since each $Z_{\xi}$ is closed. On the other hand, by $4.2, \varphi$ is continuous and satisfies $\Phi_{\xi}(\varphi)=\Phi_{\xi}\left(\varphi_{0}\right) \cup \Phi_{\xi}\left(\varphi_{1}\right)$ 
$=Z_{\xi} \cup Z_{\alpha}=Z_{\xi}$ for $\xi<\alpha$, and $\Phi_{\alpha}(\varphi)=\Phi_{\alpha}\left(\varphi_{0}\right) \cup \Phi_{\alpha}\left(\varphi_{1}\right)=\varnothing \cup Z_{\alpha}=Z_{\alpha}$. Thus, $\varphi$ satisfies 4.1(b).

Induction step: $\mu=$ limit ordinal. Since $\mu$ is countable, there is a sequence $\left\{\alpha_{i} \mid i<\omega\right\}$ of ordinal numbers such that $\alpha_{0}>0$ and $\lim _{i \rightarrow \omega} \alpha_{i}=\mu$. Choose $\left\{V_{i} \mid i<\omega\right\}$ as in 4.4. By the induction hypothesis, there exist continuous mappings $\varphi_{i}: V_{i} \rightarrow Z$ such that $\varphi_{i}\left(V_{i}^{(\xi)}\right) \subseteq Z_{\xi}$ and $\Phi_{\xi}\left(\varphi_{i}\right)=Z_{\xi}$ for all $\xi<\alpha_{i}$. Since $V_{i}^{(\xi)} \sim\left(\omega^{\alpha_{i}}\right)^{(\xi)}=\varnothing$ for $\xi \geqq \alpha_{i}$, it follows that $\varphi_{i}\left(V^{(\xi)}\right)=\varnothing$ and $\Phi_{\xi}\left(\varphi_{i}\right)=\varnothing$ for $\xi \geqq \alpha_{i}$. Let $\varphi=\bigcup_{i<\omega} \varphi_{i}$. Then $\varphi$ is continuous, and by an argument like the one given in the nonlimit case, $\varphi\left(\left(\omega^{\mu}\right)^{(\xi)}\right) \subseteq Z_{\xi}$, and $\Phi_{\xi}(\varphi)=Z_{\xi}$ for all $\xi<\mu$. Hence $\varphi$ satisfies 4.1(a) and 4.1(b).

5. Proof of 1.1. By the remarks at the end of $\S 3$, we may suppose that spaces $X$ and $Y$ in $\subseteq$ are given, together with closed subsets $Z \subseteq X$ and $W \subseteq Y$ such that $X-Z \simeq Y-W \simeq \omega^{\mu}$, and that there is a mapping $\varphi: Z \rightarrow W$ such that

$$
\varphi\left(Z \cap\left(X^{(\xi)}-Z\right)^{-}\right)=W \cap\left(Y^{(\xi)}-W\right)^{-}
$$

for all ordinal numbers $\xi$. Our objective is to extend $\varphi$ to a homeomorphism of $X$ onto $Y$. As in the previous section, it is possible to deduce Theorem 1.1 from a result concerning the mappings of $\omega^{\mu}$ into $W$.

Lemma 5.1. Let $\mu$ be a countable ordinal number larger than 0 . Let $d$ be a U.D. metric on the Boolean space $W$. Suppose that $\sigma$ and $\tau$ are continuous mappings of $\omega^{\mu}$ to $W$ satisfying

(i) $\Phi_{\xi}(\sigma)=\Phi_{\xi}(\tau)$ for all $\xi<\mu$, and

$$
\begin{aligned}
& \sup _{\xi<\mu} \sup _{\eta \in\left(\omega^{\mu}\right)(\xi)} d\left(\sigma(\eta), \Phi_{\xi}(\sigma)\right)<\delta, \\
& \sup _{\xi<\mu} \sup _{\eta \in\left(\omega^{\mu}\right)^{(\xi)}} d\left(\tau(\eta), \Phi_{\xi}(\tau)\right)<\delta .
\end{aligned}
$$

Then there is a homeomorphism $\chi$ of $\omega^{\mu}$ onto itself such that

(a) $\lim _{\eta \rightarrow \omega^{\mu}} d(\sigma(\eta), \tau(\chi(\eta)))=0$,

(b) $d(\sigma(\eta), \tau(\chi(\eta)))<2 \delta$ for all $\eta<\mu$.

The conclusion (a) is the significant part of this proposition. However, property (b) is useful in carrying out the inductive proof. Note that (ii) can always be satisfied by taking $\delta>$ diam $W$.

Before proving Lemma 5.1, let us see how it yields Theorem 1.1. If $\mu=0$, the theorem is trivial. Therefore, we can assume that $\mu \geqq 1$. Choose any U.D. metrics on $X$ and $Y$. It will cause no confusion to denote both metrics by the same letter $d$. Let $\pi$ be the nearest point retraction of $X$ onto $Z$ and denote by $\rho$ the nearest point retraction of $Y$ onto $W$. The following property of $\pi$ is needed.

(1) If $\left\{x_{i} \mid i<\omega\right\} \subseteq X-Z$ satisfies $\lim _{i \rightarrow \omega} d\left(x_{i}, Z\right)=0$, then $\lim _{i \rightarrow \omega} \pi\left(x_{i}\right)=x$ if and only if $\lim _{i \rightarrow \omega} x_{i}=x$.

Proof. Suppose that $\lim _{i \rightarrow \omega} \pi\left(x_{i}\right)=x$. Then $x \in Z$ and $d\left(x_{i}, x\right) \leqq d\left(x_{i}, \pi\left(x_{i}\right)\right)$ $+d\left(\pi\left(x_{i}\right), x\right)=d\left(x_{i}, Z\right)+d\left(\pi\left(x_{i}\right), x\right)$, which goes to zero by our hypotheses. Conversely, assume that $\lim _{i \rightarrow \omega} x_{i}=x$. Then $d(x, Z)=0$, since $\lim _{i \rightarrow \omega} d\left(x_{i}, Z\right)=0$. 
Hence, $x \in Z$ (since $Z$ is closed), and the continuity of $\pi$ yields $\lim _{i \rightarrow \omega} \pi\left(x_{i}\right)=$ $\pi(x)=x$.

It goes without saying that a similar result holds true for $\rho$.

By hypothesis, $X-Z \simeq \omega^{\mu} \simeq Y-W$. Let $\kappa: \omega^{\mu} \rightarrow X-Z$ and $\nu: \omega^{\mu} \rightarrow Y-W$ be homeomorphisms.

(2) For $\left\{\eta_{i} \mid i<\omega\right\} \subseteq \omega^{\mu}$, the conditions $\lim _{i \rightarrow \omega} \eta_{i}=\omega^{\mu}$ and $\lim _{i \rightarrow \omega} d\left(\kappa\left(\eta_{i}\right), Z\right)=0$ (or $\lim _{i \rightarrow \omega} d\left(\nu\left(\eta_{i}\right), W\right)=0$ ) are equivalent.

Proof. The two conditions are equivalent to the statement that no compact subset (of $\omega^{\mu}$ or $X-Z$ ) contains more than a finite number of elements from the respective sequences. This property is clearly preserved by a homeomorphism.

Using (1) and (2), we can now prove

(3) $\Phi_{\xi}(\pi \kappa)=Z \cap\left(X^{(\xi)}-Z\right)^{-}$and $\Phi_{\xi}(\rho \nu)=W \cap\left(Y^{(\xi)}-W\right)^{-}$for all $\xi<\mu$.

Proof. By definition, $x \in \Phi_{\xi}(\pi \kappa)$ if and only if there is a sequence $\left\{\eta_{i} \mid i<\omega\right\}$ $\subseteq\left(\omega^{\mu}\right)^{(\xi)}$ such that $\lim _{i \rightarrow \omega} \eta_{i}=\omega^{\mu}$, and $\lim _{i \rightarrow \omega} \pi \kappa\left(\eta_{i}\right)=x$. By virtue of (1) and (2), this in turn is equivalent to the existence of a sequence $\left\{x_{i} \mid i<\omega\right\} \subseteq X^{(\xi)}-Z$ which converges to $x$, together with the condition $x \in Z$. Thus, we see that $\Phi_{\xi}(\pi \kappa)$ $=Z \cap\left(X^{(\xi)}-Z\right)^{-}$. Similarly, $\Phi_{\xi}(\rho \nu)=W \cap\left(X^{(\xi)}-W\right)^{-}$.

Define $\sigma=\varphi \pi \kappa: \omega^{\mu} \rightarrow W$ and $\tau=\rho \nu: \omega^{\mu} \rightarrow W$. Then

$$
\begin{aligned}
\Phi_{\xi}(\sigma) & =\Phi_{\xi}(\varphi(\pi \kappa))=\bigcap_{\eta<\omega^{\mu}}\left((\varphi \pi \kappa)\left(\left(\omega^{\mu}\right)^{(\xi)} \cap\left[\eta, \omega^{\mu}\right)\right)\right)^{-} \\
& =\varphi\left(\bigcap_{\eta<\omega^{\mu}}\left((\pi \kappa)\left(\left(\omega^{\mu}\right)^{(\xi)} \cap\left[\eta, \omega^{\mu}\right)\right)\right)^{-}\right) \\
& =\varphi\left(\Phi_{\xi}(\pi \kappa)\right)=\varphi\left(Z \cap\left(X^{(\xi)}-Z\right)^{-}\right)
\end{aligned}
$$

by (3). By hypothesis, $\left(Z \cap\left(X^{(\xi)}-Z\right)^{-}=W \cap\left(Y^{(\xi)}-W\right)^{-}\right.$so that by (3) again we obtain $\Phi_{\xi}(\sigma)=W \cap\left(Y^{(\xi)}-W\right)^{-}=\Phi_{\xi}(\tau)$. Therefore, the hypothesis (i) of 5.1 is satisfied. Hence, there is a homeomorphism $\chi: \omega^{\mu} \rightarrow \omega^{\mu}$ such that

(4) $\lim _{\eta \rightarrow \omega^{\mu}} d(\sigma(\eta), \tau(\chi(\eta)))=0$.

Finally, define $\psi: X \rightarrow Y$ by

$$
\begin{array}{ll}
\psi(x)=\nu\left(\chi\left(\kappa^{-1}(x)\right)\right) & \text { if } x \in X-Z, \\
\psi(x)=\varphi(x) & \text { if } x \in Z .
\end{array}
$$

Plainly, $\psi$ is bijective and $\psi \mid Z=\varphi$. Since $X$ is compact, it suffices to show that $\psi$ is continuous. Continuity for $\psi$ easily reduces to proving the following fact.

(5) If $\left\{x_{i} \mid i<\omega\right\} \subseteq X-Z$, and $\lim _{i \rightarrow \omega} x_{i}=x \in Z$, then $\lim _{i \rightarrow \omega} \psi\left(x_{i}\right)=\varphi(x)$.

Proof. By (2), $\lim _{i \rightarrow \omega} \kappa^{-1}\left(x_{i}\right)=\omega^{\mu}$. By continuity,

$$
\lim _{i \rightarrow \omega} \sigma\left(\kappa^{-1}\left(x_{i}\right)\right)=\lim _{i \rightarrow \omega} \varphi \pi \kappa\left(\kappa^{-1}\left(x_{i}\right)\right)=\varphi \pi\left(\lim _{i \rightarrow \omega} x_{i}\right)=\varphi(\pi(x))=\varphi(x)
$$

(since $x \in Z$ ). Combining these observations with (4), we obtain

$$
\lim _{i \rightarrow \omega} \rho\left(\varphi\left(x_{i}\right)\right)=\lim _{i \rightarrow \omega} \rho \nu \chi^{-1}\left(x_{i}\right)=\lim _{i \rightarrow \omega} \tau \chi\left(\kappa^{-1}\left(x_{i}\right)\right)=\varphi(x) .
$$

Since $\chi$ is a homeomorphism, $\lim _{i \rightarrow \omega} \kappa^{-1}\left(x_{i}\right)=\omega^{\mu}$ implies $\lim _{i \rightarrow \omega} \chi\left(\kappa^{-1}\left(x_{i}\right)\right)=\omega^{\mu}$. 
Thus, by (2), $\lim _{i \rightarrow \omega} d\left(\varphi\left(x_{i}\right), W\right)=\lim _{i \rightarrow \omega} d\left(\nu \chi^{\kappa}{ }^{-1}\left(x_{i}\right), W\right)=0$. Hence, the analogue for $\rho$ of (1), applied to $\lim _{i \rightarrow \omega} \rho\left(\varphi\left(x_{i}\right)\right)=\varphi(x)$, gives the desired result $\lim _{i \rightarrow \omega} \psi\left(x_{i}\right)$ $=\varphi(x)$. This completes the deduction of 1.1 from 5.1.

We turn now to the task of proving 5.1 by transfinite induction on $\mu$. The following notation will be useful. Let $F$ and $G$ be subsets of a compact metric space, such that $F^{-} \supseteq G$. Denote $e(F, G)=\sup _{x \in F} d(x, G)$.

The following properties of $e$ are easily obtained from this definition:

(a) $e(F, G)<\varepsilon$ implies that for every $x \in F$, there exists $y \in G$ such that $d(x, y)<\varepsilon$; if $F$ is closed, the converse is true;

(b) $e(F, G)=e\left(F^{-}, G^{-}\right)$;

(c) $F^{-} \supseteq G$ and $G^{-} \supseteq H$ implies $e(F, G) \leqq e(F, H) \leqq e(F, G)+e(G, H)$;

(d) $e(F, F)=0$;

(e) if $V$ is an ordered set and $\left\{F_{v} \mid v \in V\right\}$ is a nested family of closed subsets of a compact metric space (i.e., $v<v^{\prime}$ in $V$ implies $\left.F_{v} \supseteq F_{v^{\prime}}\right)$, then $\lim _{v \rightarrow \infty} e\left(F_{v}, \bigcap_{u \in V} F_{u}\right)$ $=0$.

Suppose now that $\varphi$ is a continuous mapping of $\omega^{\mu}$ into $W$. Denote

$$
r_{\xi}(\varphi)=e\left(\varphi\left(\left(\omega^{\mu}\right)^{(\xi)}\right), \Phi_{\xi}(\varphi)\right), \quad r(\varphi)=\sup _{\xi<\mu} r_{\xi}(\varphi) .
$$

More generally, if $V$ is an open subset of $\omega^{\mu}$, denote

$$
r_{\xi}(\varphi, V)=e\left(\varphi\left(V \cap\left(\omega^{\mu}\right)^{(\xi)}\right), \Phi_{\xi}(\varphi \mid V)\right), \quad r(\varphi, V)=\sup _{\xi<\mu} r_{\xi}(\varphi, V) .
$$

Using this notation, the condition (ii) of 5.1 can be reformulated more simply as $r(\sigma)<\delta$ and $r(\tau)<\delta$.

Case : $\mu=1$. Since the order topology on $\omega$ is discrete, continuity is no restriction in this case. The first step of the argument is a proof that conclusion of 5.1 holds when the requirement that $\chi$ be surjective is dropped. The full statement of the proposition is then obtained by a modification of the usual method used to prove the Schröder-Bernstein theorem.

(6) Under the hypotheses of 5.1 , with $\mu=1$, there exists a strictly increasing mapping $\pi: \omega \rightarrow \omega$ such that

(a) $\lim _{\eta \rightarrow \omega} d(\sigma(\eta), \tau(\pi(\eta)))=0$

(b) $d(\sigma(\eta), \tau(\pi(\eta)))<2 \delta$ for all $\eta<\omega$, and

(c) $\pi(0)>0$.

Proof. For $\eta<\omega$, choose $x_{\eta}$ to be the unique point of $\Phi_{0}(\sigma)$ which is nearest to $\sigma(\eta)$. Note that $\lim _{\eta \rightarrow \omega} d\left(\sigma(\eta), x_{\eta}\right)=0$, and $d\left(\sigma(\eta), x_{\eta}\right)<\delta$ for all $\eta<\omega$. Let $\varepsilon_{0}>\varepsilon_{1}$ $>\varepsilon_{2}>\cdots$ be a sequence of positive real numbers with $\varepsilon_{0}<\delta$ and $\lim _{\eta \rightarrow \omega} \varepsilon_{\eta}=0$. Define $\pi(\eta)$ by recursion so that it satisfies $0<\pi(0)<\pi(1)<\cdots<\pi(\eta)<\pi(\eta+1)$ $<\cdots$, and

$$
d\left(x_{\eta}, \tau(\pi(\eta))\right) \leqq \varepsilon_{\eta}, \text { for all } \eta<\omega .
$$

These conditions can be satisfied, because $x_{\eta} \in \Phi_{0}(\sigma)=\Phi_{0}(\tau)$ implies that there is a subsequence $\left\{\eta_{i} \mid i<\omega\right\} \subseteq \omega$ such that $\lim _{i \rightarrow \omega} \tau\left(\eta_{i}\right)=x_{\eta}$. Since $d(\sigma(\eta), \tau(\pi(\eta)))$ $\leqq d\left(\sigma(\eta), x_{\eta}\right)+d\left(x_{\eta}, \tau(\pi(\eta))\right) \leqq d\left(\sigma(\eta), x_{\eta}\right)+\varepsilon_{\eta}$, it is clear that $\pi$ satisfies (a) and (b). 
In order to complete the proof of 5.1 in the case $\mu=1$, let $\pi: \omega \rightarrow \omega$ be strictly increasing, and satisfy conditions (a)-(c) of (6). Similarly, let $\rho: \omega \rightarrow \omega$ be strictly increasing, and such that $\lim _{\eta \rightarrow \omega} d(\tau(\eta), \sigma(\rho(\eta)))=0, d(\tau(\eta), \sigma(\rho(\eta)))<2 \delta$ for all $\eta<\omega$, and $\rho(0)>0$. By recursion, define

$$
K_{0}=L_{0}=\omega, \quad K_{n}=\rho\left(L_{n-1}\right), \quad L_{n}=\pi\left(K_{n-1}\right), \quad \text { for } n \geqq 1 .
$$

Note that $K_{0} \supseteq K_{1} \supseteq K_{2} \supseteq \cdots, L_{0} \supseteq L_{1} \supseteq L_{2} \supseteq \cdots$, and

$$
K_{2 n}=(\rho \pi)^{n}(\omega), \quad L_{2 n}=(\tau \rho)^{n}(\omega) .
$$

This last observation yields the conclusions

$$
K_{2 n} \subseteq\{k<\omega \mid k \geqq 2 n\} \text { and } L_{2 n} \subseteq\{k<\omega \mid k \geqq 2 n\} .
$$

In fact, since $\pi$ and $\rho$ are strictly increasing, and $\pi(0)>0, \rho(0)>0$, it follows that $\pi(k)>k$ and $\rho(k)>k$ for all $k$. Therefore, $(\rho \pi)^{n}(k) \geqq k+2 n$ and $(\pi \rho)^{n}(k) \geqq k+2 n$ by iteration. It follows that $\bigcap_{n<\omega} K_{n}=\bigcap_{n<\omega} L_{n}=\varnothing$. Hence, we have disjoint decompositions

$$
\omega=\bigcup_{n<\omega}\left(K_{n}-K_{n+1}\right)=\bigcup_{n<\omega}\left(L_{n}-L_{n+1}\right) .
$$

Since $\pi$ and $\rho$ are one-to-one mappings, it follows that $\pi\left(K_{n}-K_{n+1}\right)=L_{n+1}-L_{n+2}$, and $\rho\left(L_{n}-L_{n+1}\right)=K_{n+1}-K_{n+2}$. Define $\chi: \omega \rightarrow \omega$ by

$$
\begin{array}{ll}
\chi(\eta)=\pi(\eta) & \text { for } \eta \in \bigcup_{m<\omega}\left(K_{2 m}-K_{2 m+1}\right), \\
\chi(\eta)=\left(\rho^{-1}\right)(\eta) & \text { for } \eta \in \bigcup_{m<\omega}\left(K_{2 m+1}-K_{2 m+2}\right) .
\end{array}
$$

Plainly, $\chi$ is a well-defined, bijective mapping of $\omega$ onto $\omega$. If $\eta \in K_{2 m}-K_{2 m+1}$, then $d(\sigma(\eta), \tau(\chi(\eta)))=d(\sigma(\eta), \tau(\pi(\eta)))<2 \delta$. If $\eta \in K_{2 m+1}-K_{2 m+2}$, then $\eta=\rho(\zeta)$ for some $\zeta \in L_{2 m}-L_{2 m+1}$, so that $d(\sigma(\eta), \tau(\chi(\eta)))=d(\sigma(\rho(\xi)), \tau(\xi))<2 \delta$. From these observations, it is also clear that $\lim _{\eta \rightarrow \omega} d(\sigma(\eta), \tau(\chi(\eta)))=0$. Therefore, 5.1 holds when $\mu=1$.

Induction step: $\mu=\alpha+1$. By the results of $\$ 4$ in [3], $\left(\omega^{\mu}\right)^{(\alpha)}=\left\{\omega^{\alpha} \cdot n \mid 0<n<\omega\right\}$. As we have just shown, there is a one-to-one mapping $\left({ }^{1}\right) \pi$ of $\omega$ onto itself such that

(7) $\lim _{n \rightarrow \omega} d\left(\sigma\left(\omega^{\alpha} \cdot n\right), \tau\left(\omega^{\alpha} \cdot \pi(n)\right)\right)=0$, and

(8) $d\left(\sigma\left(\omega^{\alpha} \cdot n\right), \tau\left(\omega^{\alpha} \cdot \pi(n)\right)\right)<2 \delta$ for $0<n<\omega$.

From these two properties, it follows that

(9) $\varepsilon=2 \delta-\left(\sup _{0<n<\omega} d\left(\sigma\left(\omega^{\alpha} \cdot n\right), \tau\left(\omega^{\alpha} \cdot \pi(n)\right)\right)\right)>0$.

Let $\varepsilon_{1}>\varepsilon_{2}>\varepsilon>\cdots$ be a sequence of real numbers such that $\varepsilon_{1}=\varepsilon / 2$ (where $\varepsilon$ is defined in (9)), and $\lim _{n \rightarrow \omega} \varepsilon_{n}=0$. By the continuity of $\sigma$ and $\tau$, there exists an increasing sequence $\left\{\alpha_{n} \mid 0 \leqq n<\omega\right\} \subseteq \omega^{\alpha}$ satisfying

(10) $\lim _{n \rightarrow \omega} \alpha_{n}=\omega^{\alpha}$,

(1) The mapping $\pi$ in this part of the proof coincides with the $\chi$ that was constructed in the proof of the case $\mu=1$. It should not be confused with the mapping $\pi$ that was obtained in (6). 
(11) if $\omega^{\alpha} \cdot(n-1)+\alpha_{n} \leqq \eta \leqq \omega^{\alpha} \cdot n$, then $d\left(\sigma(\eta), \sigma\left(\omega^{\alpha} \cdot n\right)\right)<\varepsilon_{n}$, and

(12) if $\omega^{\alpha} \cdot(\pi(n)-1)+\alpha_{n} \leqq \eta \leqq \omega^{\alpha} \cdot \pi(n)$, then $d\left(\tau(\eta), \tau\left(\omega^{\alpha} \cdot \pi(n)\right)\right)<\varepsilon_{n}$.

Next, choose an increasing sequence $\left\{\beta_{n} \mid 0<n<\omega\right\} \subseteq \omega^{\alpha}$ satisfying either

(13) $\beta_{n} \geqq \alpha_{n}+\omega^{\gamma}$ if $\alpha=\gamma+1$,

or, when $\alpha$ is a limit ordinal,

(13') $\beta_{n} \geqq \alpha_{n}+\omega^{\gamma_{n}}$ for some sequence $\gamma_{1}<\gamma_{2}<\cdots$, with $\lim _{n \rightarrow \omega} \gamma_{n}=\alpha$.

By (10), $\lim _{n \rightarrow \omega} \beta_{n}=\omega^{\alpha}$, and since $\beta_{n}<\omega^{\alpha}$ for all $n<\omega$, it follows that

(14) $\sum_{0<n<\omega} \beta_{n}=\omega^{\alpha}$ and $\sum_{0<n<\omega} \beta_{\pi(n)}=\omega^{\alpha}$.

Define subsets $U_{0}, V_{0}, U_{1}$, and $V_{1}$ of $\omega^{\alpha}$ as follows:

$$
\begin{aligned}
& U_{0}=\left[0, \beta_{1}\right] \cup \bigcup_{0<n<\omega}\left(\omega^{\alpha} \cdot n, \omega^{\alpha} \cdot n+\beta_{n+1}\right], \\
& V_{0}=\left[0, \beta_{1}\right] \cup \bigcup_{0<n<\omega}\left(\omega^{\alpha} \cdot \pi(n), \omega^{\alpha} \cdot \pi(n)+\beta_{n+1}\right], \\
& U_{1}=\omega^{\mu}-U_{0}=\bigcup_{0<n<\omega}\left(\omega^{\alpha} \cdot(n-1)+\beta_{n}, \omega^{\alpha} \cdot n\right], \\
& V_{1}=\omega^{\mu}-V_{0}=\bigcup_{0<n<\omega}\left(\omega^{\alpha} \cdot(\pi(n)-1)+\beta_{n}, \omega^{\alpha} \cdot \pi(n)\right] .
\end{aligned}
$$

Clearly, $U_{0}, V_{0}, U_{1}$, and $V_{1}$ are open and closed subsets of $\omega^{\mu}$. By virtue of (14), $U_{0}$ and $V_{0}$ are order isomorphic to $\omega^{\alpha}$. Also, because of (13), we have in the case that $\alpha=\gamma+1$,

$$
\begin{aligned}
& U_{0} \cap\left(\omega^{\mu}\right)^{(\gamma)} \cap\left[\omega^{\alpha} \cdot n+\alpha_{n+1}, \omega^{\alpha} \cdot n+\beta_{n+1}\right] \neq \varnothing, \text { and } \\
& V_{0} \cap\left(\omega^{\mu}\right)^{(\gamma)} \cap\left[\omega^{\alpha} \cdot \pi(n)+\alpha_{n+1}, \omega^{\alpha} \cdot \pi(n)+\beta_{n+1}\right] \neq \varnothing .
\end{aligned}
$$

Similarly, (13') implies that if $\alpha$ is a limit ordinal, then

$$
\begin{aligned}
& U_{0} \cap\left(\omega^{\mu}\right)^{\left(\gamma_{m}\right)} \cap\left[\omega^{\alpha} \cdot n+\alpha_{n+1}, \omega^{\alpha} \cdot n+\beta_{n+1}\right] \neq \varnothing, \text { and } \\
& V_{0} \cap\left(\omega^{\mu}\right)^{\left(\gamma_{m}\right)} \cap\left[\omega^{\alpha} \cdot \pi(n)+\alpha_{n+1}, \omega^{\alpha} \cdot \pi(n)+\beta_{n+1}\right] \neq \varnothing,
\end{aligned}
$$

provided $m \leqq n+1$. Define a mapping $\chi_{1}: U_{1} \rightarrow V_{1}$ by

$$
\chi_{1}\left(\omega^{\alpha} \cdot(n-1)+\zeta\right)=\omega^{\alpha}(\pi(n)-1)+\zeta, \quad \beta_{n}<\zeta \leqq \omega^{\alpha} .
$$

Plainly, $\chi_{1}$ is a homeomorphism of $U_{1}$ onto $V_{1}$. Moreover, if $\beta_{n}<\zeta \leqq \omega^{\alpha}$, then by (11), (12), and (13),

$$
\begin{aligned}
d\left(\sigma\left(\omega^{\alpha} \cdot(n-1)+\zeta\right), \tau\left(\chi_{1}\left(\omega^{\alpha} \cdot(n-1)+\zeta\right)\right)\right) & =d\left(\sigma\left(\omega^{\alpha} \cdot(n-1)+\zeta\right), \tau\left(\omega^{\alpha}(\pi(n)-1)+\zeta\right)\right) \\
& <d\left(\sigma\left(\omega^{\alpha} \cdot n\right), \tau\left(\omega^{\alpha} \cdot \pi(n)\right)\right)+2 \varepsilon_{n} .
\end{aligned}
$$

Therefore, by (7) and (9), $\lim _{\eta \rightarrow \omega^{u}, \eta \in U_{1}} d\left(\sigma(\eta), \tau\left(\chi_{1}(\eta)\right)\right)=0$, and

$$
d\left(\sigma(\eta), \tau\left(\chi_{1}(\eta)\right)\right)<2 \delta \text { for all } \eta \in U_{1} .
$$

We complete the proof of this part of the induction by showing that the induction hypothesis can be applied to $U_{0}$ and $V_{0}$. It is enough to prove

(16) $\Phi_{\xi}\left(\sigma \mid U_{0}\right)=\Phi_{\xi}(\sigma)$, and $\Phi_{\xi}\left(\tau \mid V_{0}\right)=\Phi_{\xi}(\tau)$ for all $\xi<\alpha$.

Indeed, it will follow from (16) that $r\left(\sigma, U_{0}\right) \leqq r(\sigma)<\delta$, and $r\left(\tau, V_{0}\right)<\delta$, so that since $U_{0} \sim \omega^{\alpha} \sim V_{0}$, the induction hypothesis yields a homeomorphism $\chi_{0}$ of $U_{0}$ 
onto $V_{0}$ such that $\lim _{\eta \rightarrow \omega^{\mu}, \eta \in U_{0}} d\left(\sigma(\eta), \tau\left(\chi_{0}(\eta)\right)\right)=0$ and $d\left(\sigma(\eta), \tau\left(\chi_{0}(\eta)\right)\right)<2 \delta$ for all $\eta \in U_{0}$. Then $\chi=\chi_{0} \cup \chi_{1}$ is a homeomorphism of $\omega^{\mu}$ onto itself satisfying (a) and (b) of 5.1 .

Proof of (16). Since $U_{0}$ is open and cofinal in $\omega^{\mu}$, it follows from Lemma 2.1 that $\Phi_{\xi}\left(\sigma \mid U_{0}\right) \subseteq \Phi_{\xi}(\sigma)$. Suppose that $x \in \Phi_{\xi}(\sigma)$. Let $\left\{\eta_{n} \mid n<\omega\right\}$ be a sequence in $\left(\omega^{\mu}\right)^{(\xi)}$ such that $\lim _{n \rightarrow \omega} \eta_{n}=\omega^{\mu}$ and $\lim _{n \rightarrow \omega} \sigma\left(\eta_{n}\right)=x$. Without loss of generality, we can assume $\eta_{n} \geqq \omega^{\alpha} \cdot(n-1)+\alpha_{n}$. Let $m<\omega$ be defined by $m=0$ if $\alpha$ is a nonlimit ordinal, and if $\alpha$ is a limit ordinal, then take $m$ to be the smallest number such that $\gamma_{m} \geqq \xi$, where $\gamma_{1}, \gamma_{2}, \ldots$ is the sequence which was chosen in (13'). Since $\xi<\alpha, m$ is well defined. For $n>m$, define $\xi_{n}$ by the conditions

$$
\begin{aligned}
\zeta_{n}=\eta_{n} \quad \text { if } \eta_{n} \in U_{0}, \\
\zeta_{n}=\min \left(U_{0} \cap\left(\omega^{\alpha}\right)^{(\xi)} \cap\left[\omega^{\alpha} \cdot(k-1)+\alpha_{k}, \omega^{\alpha} \cdot(k-1)+\beta_{k}\right]\right) \\
\text { if } \eta_{n} \in\left(\omega^{\alpha} \cdot(k-1)+\beta_{k}, \omega^{\alpha} \cdot k\right) .
\end{aligned}
$$

This definition makes sense by virtue of $(15)$ or $\left(15^{\prime}\right)$, and the fact that $\eta_{n}>$ $\omega^{\alpha} \cdot(n-1)+\alpha_{n}$ implies $k \geqq n>m$ and $\gamma_{k-1} \geqq \gamma_{m} \geqq \xi$ when $\eta_{n} \notin U_{0}$. By definition of $\zeta_{n}$, we have $d\left(\sigma\left(\eta_{n}\right), \sigma\left(\zeta_{n}\right)\right)<2 \varepsilon_{k} \leqq 2 \varepsilon_{n}$ in all cases. Also, $\zeta_{n} \in U_{0} \cap\left(\omega^{\mu}\right)^{(\xi)} \cap\left[\omega^{\alpha} \cdot(n-1)\right.$ $\left.+\alpha_{n}, \omega^{\mu}\right)$. Hence, $\lim _{n \rightarrow \omega} \zeta_{n}=\omega^{\mu}$ and $\lim _{n \rightarrow \omega} \sigma\left(\zeta_{n}\right)=\lim _{n \rightarrow \omega} \sigma\left(\eta_{n}\right)=x$. This proves that $x \in \Phi_{\xi}\left(\sigma \mid U_{0}\right)$. Consequently, $\Phi_{\xi}\left(\sigma \mid U_{0}\right)=\Phi_{\xi}(\sigma)$. The proof that $\Phi_{\xi}\left(\tau \mid V_{0}\right)$ $=\Phi_{\xi}(\tau)$ is similar.

Induction step: $\mu=$ limit ordinal. Since the basic idea of the proof in this case may be obscured by technical details, it seems worthwhile to begin with an outline of the argument. The essential idea is to reduce the proof to the special case of 5.1 in which there exists an ordinal number $\alpha<\mu$ such that $\Phi_{\xi}(\sigma)=\Phi_{\xi}(\tau)=\left\{x_{0}\right\}$ for all $\xi>\alpha$. This reduction will be accomplished by the following lemma.

Lemma 5.2. Assume that the hypotheses of 5.1 are satisfied with $\mu$ a limit ordinal. Let $\beta_{0}<\beta_{1}<\beta_{2}<\cdots$ be a sequence of ordinal numbers such that $\lim _{n \rightarrow \omega} \beta_{n}=\mu$, and let $\delta_{0} \geqq \delta_{1} \geqq \delta_{2} \geqq \cdots$ be a sequence of positive real numbers such that $\delta_{0} \leqq \delta$ and $\lim _{n \rightarrow \omega} \delta_{n}=0$. Choose any $x_{0} \in \bigcap_{\xi<\mu} \Phi_{\xi}(\sigma)$. Then there exists a strictly increasing sequence $\left\{\alpha_{n} \mid n<\omega\right\}$ of ordinal numbers, and families $\left\{U_{n} \mid n<\omega\right\},\left\{V_{n} \mid n<\omega\right\}$ of open and closed subsets of $\omega^{\mu}$ such that
(a) $\beta_{n}<\alpha_{n}<\mu$,
(b) $U_{n} \cup V_{n} \subseteq\left(\omega^{\beta_{n-1}}, \omega^{\mu}\right)$ if $n>0$,
(c) $U_{n} \cap U_{m}=V_{n} \cap V_{m}=\varnothing$ if $m \neq n$,
(d) $\bigcup_{n<\omega} U_{n}=\bigcup_{n<\omega} V_{n}=\omega^{\mu}$,
(e) $U_{n} \cap\left(\omega^{\mu}\right)^{(\xi)}$ and $V_{n} \cap\left(\omega^{\mu}\right)^{(\xi)}$ are cofinal in $\omega^{\mu}$ for every $\xi<\mu$,
(f) $r\left(\sigma, U_{n}\right)<\delta_{n}, r\left(\tau, V_{n}\right)<\delta_{n}$ for all $n<\omega$,
(g) $\Phi_{\xi}\left(\sigma \mid U_{n}\right)=\Phi_{\xi}\left(\tau \mid V_{n}\right)=\left\{x_{0}\right\}$ if $\xi>\alpha_{n}$,
(h) $\Phi_{\xi}\left(\sigma \mid U_{n}\right)=\Phi_{\xi}\left(\tau \mid V_{n}\right)=\Phi_{\xi}(\sigma)$ if $n>0$ and $\alpha_{n-1}<\xi \leqq \alpha_{n}$, or $n=0$ and $\xi \leqq \alpha_{0}$,
(i) $\Phi_{\xi}\left(\sigma \mid U_{n}\right)=\Phi_{\xi}\left(\tau \mid V_{n}\right)=\Phi_{\alpha_{n-1}+1}(\sigma)$ if $n>0$ and $\xi \leqq \alpha_{n-1}$. 
It is easy to deduce from (e) that $U_{n} \sim \omega^{\mu}$ and $V_{n} \sim \omega^{\mu}$. Thus, the special case of 5.1 can be used to define a homeomorphism $\chi_{n}: U_{n} \rightarrow V_{n}$ such that

$$
\lim _{n \rightarrow \omega^{\mu}, \eta \in U_{n}} d\left(\sigma(\eta), \tau\left(\chi_{n}(\eta)\right)\right)=0
$$

and $d\left(\sigma(\eta), \tau\left(\chi_{n}(\eta)\right)\right)<\delta_{n}$ for all $\eta \in U_{n}$. It then follows without difficulty that $\chi=\bigcup_{n<\omega} \chi_{n}$ satisfies the conditions of 5.1.

We begin with some lemmas which lead to a proof of the special case of 5.1. The first two of these results do not require the hypothesis that $\mu$ is a limit ordinal.

LEMMA 5.3. Let $0<\xi<\mu$, and suppose that for each $\eta \in\left(\omega^{\mu}\right)^{(\xi)}$, there is given $\zeta_{\eta}<\eta$. Define $V=\bigcup_{\eta \in\left(\omega^{\mu}\right)^{(\xi)}}\left(\zeta_{\eta}, \eta\right]$.

Then $V$ is open and closed in $\omega^{\mu}$, and $V \supseteq\left(\omega^{\mu}\right)^{(\xi)}$.

Proof. Plainly, $V$ is open and $V \supseteq\left(\omega^{\mu}\right)^{(\xi)}$. Suppose that $\rho \in \omega^{\mu}-V$. Let $\eta$ be the smallest member of $\left(\omega^{\mu}\right)^{(\xi)} \cap\left(\rho, \omega^{\mu}\right]$. Note that $\eta \notin\left(\omega^{\mu}\right)^{(\xi+1)}$, since otherwise there exists $\eta^{\prime} \in\left(\omega^{\mu}\right)^{(\xi)}$ such that $\rho<\eta^{\prime}<\eta$. Thus, either $\eta$ is the smallest element of $\left(\omega^{\mu}\right)^{(\xi)}$, or else $\eta$ has a predecessor $\eta_{1}$ in $\left(\omega^{\mu}\right)^{(\xi)}$. Plainly, $\eta_{1}<\rho$. Since $\rho \leqq \zeta_{\eta}$ (otherwise, $\left.\rho \in\left(\zeta_{\eta}, \eta\right] \subseteq V\right)$, it follows that either $\left[0, \zeta_{n}\right]$, or $\left(\eta_{1}, \zeta_{n}\right]$ is a neighborhood of $p$, contained in $\omega^{\mu}-V$. Hence, $V$ is closed.

Lemma 5.4. Let $V$ be a subset of $\omega^{\mu}$ which is open and closed. Suppose that $\alpha \leqq \mu$, and $V$ satisfies $V \cap\left(\omega^{\mu}\right)^{(\alpha)}=\varnothing$, and $V \cap\left(\omega^{\mu}\right)^{(\xi)}$ is cofinal in $\omega^{\mu}$ for every $\xi<\alpha$. Then $V$ is order isomorphic to $\omega^{\alpha}$.

Proof. Since $V$ is well ordered, there is an order isomorphism of $V$ onto an ordinal number $\zeta$. Because $V$ is closed, its order topology coincides with its relative topology. Hence, by Lemma 2.1 and our hypotheses, $\zeta$ (considered as a topological space with its order topology) satisfies: $\zeta^{(\alpha)}=\varnothing$, and if $\xi<\alpha$, then $\zeta^{(\xi)} \neq \varnothing$, and $\zeta^{(\xi)}$ has no last element.

Let $\zeta=\omega^{\gamma_{1}}: n_{1}+\omega^{\gamma_{2}} \cdot n_{2}+\cdots+\omega^{\gamma_{m}} \cdot n_{m}$, where $\zeta \geqq \gamma_{1}>\gamma_{2}>\cdots>\gamma_{m}$ and $0<n_{i}<\omega$ for all $i$ (see [2, p. 67]). If either $n_{m}>1$, or $m>1$, then it follows from the results of $\S 4$ in [3] that $\zeta^{\left(\gamma_{m}\right)}$ has a largest element, namely $\omega^{\gamma_{1}} \cdot n_{1}+\omega^{\gamma_{2}} \cdot n_{2}+\cdots+\omega^{\gamma_{m}} \cdot\left(n_{m}-1\right)$. Thus, $\zeta=\omega^{\gamma_{1}}$. The conditions $\zeta^{(\xi)} \neq \varnothing$ for $\xi<\alpha$ and $\zeta^{(\alpha)}=\varnothing$ imply that $\gamma_{1}=\alpha$.

It will be convenient to call a subset $V$ of a well ordered set $U$ full in $U$ if $V$ is open and closed in $U$, and $V \cap U^{(\xi)}=V^{(\xi)}$ is cofinal in $U$ for every $\xi<\lambda(U)$. By 5.4 , if $V$ is full in $\omega^{\mu}$, then $V \sim \omega^{\mu}$.

LEMMA 5.5. Let $\mu$ be a countable limit ordinal, $\alpha<\mu, \sigma: \omega^{\mu} \rightarrow W$ a continuous mapping, and $U$ a full subset of $\omega^{\mu}$. Then there exists a strictly increasing, cofinal sequence $\left\{\eta_{n} \mid n<\omega\right\} \subseteq\left(\left(\omega^{\mu}\right)^{(\alpha)} \cap U\right)-\left(\omega^{\mu}\right)^{(\alpha+1)}$ such that

$$
\Phi_{\alpha}(\sigma \mid U)=\bigcap_{m<\omega}\left(\sigma\left(\left\{\eta_{n} \mid n \geqq m\right\}\right)\right)^{-} .
$$

Proof. Let $\left\{x_{k} \mid k<\omega\right\}$ be a countable, dense subset of $\Phi_{\alpha}(\sigma \mid U)$. Choose a sequence $\left\{\varepsilon_{m} \mid m<\omega\right\}$ of positive real numbers such that $\lim _{m \rightarrow \omega} \varepsilon_{m}=0$. Let 
$\left\{\beta_{n} \mid n<\omega\right\}$ be a strictly increasing cofinal subsequence of $\mu$. Finally, take $\pi$ to be any mapping of $\omega$ onto $\omega \times \omega$. Define $\eta_{n}$ recursively by the conditions

(i) if $\pi(n)=(k, m)$, then $d\left(\sigma\left(\eta_{n}\right), x_{k}\right)<\varepsilon_{m}$,

(ii) $\eta_{n}>\omega^{\beta_{n}}$,

(iii) if $n>0$, then $\eta_{n}>\eta_{n-1}$,

(iv) $\eta_{n}$ is the smallest ordinal number in $\left(\omega^{\mu}\right)^{(\alpha)} \cap U$ which satisfies (i), (ii), and (iii).

It is evident from the definition of $\Phi_{\alpha}(\sigma \mid U)$ that the set of $\eta \in\left(\omega^{\mu}\right)^{(\alpha)} \cap U$ satisfying (i), (ii), and (iii) is nonempty, so that the definition of $\eta_{n}$ is well posed. We must first show that $\eta_{n} \notin\left(\omega^{\mu}\right)^{(\alpha+1)}$. Suppose otherwise. Then using the continuity of $\sigma$ and the fact that $U$ is open, it would be possible to find $\eta \in\left(\omega^{\mu}\right)^{(\alpha)} \cap U$ such that

$$
d\left(\sigma\left(\eta_{n}\right), \sigma(\eta)\right)<\varepsilon_{m}-d\left(\sigma\left(\eta_{n}\right), x_{k}\right) \text { and } \eta_{n}>\eta>\max \left\{\omega^{\beta_{n}}, \eta_{n-1}\right\} .
$$

However, this would plainly contradict the minimality of $\eta_{n}$. It follows from (iii) and (ii) that the sequence $\left\{\eta_{n} \mid n<\omega\right\}$ is strictly increasing and cofinal in $\omega^{\mu}$. From (i) and the fact that $\pi$ maps $\omega$ onto $\omega \times \omega$, we conclude that for any $m<\omega$,

$$
\left\{\sigma\left(\eta_{n}\right) \mid n \geqq m\right\}^{-} \supseteq\left\{x_{k} \mid k<\omega\right\}^{-}=\Phi_{\alpha}(\sigma \mid U) .
$$

On the other hand,

$$
\bigcap_{n<\omega}\left(\sigma\left(\left(\omega^{\mu}\right)^{(\alpha)} \cap U \cap\left[\omega^{\beta_{n}}, \omega^{\mu}\right)\right)\right)^{-}=\Phi_{\alpha}(\sigma \mid U),
$$

so that by compactness, if $\varepsilon>0$, then $\left\{n \mid d\left(\sigma\left(\eta_{n}\right), \Phi_{\alpha}(\sigma \mid U)\right) \geqq \varepsilon\right\}$ is finite. Thus, $\bigcap_{m<\omega}\left(\sigma\left(\left\{\eta_{n} \mid n \geqq m\right\}\right)\right)^{-}=\Phi_{\alpha}(\sigma \mid U)$.

We will now use Lemmas 5.2-5.5 to complete the inductive proof of 5.1. The somewhat tedious proof of 5.2 is postponed to the end of the section.

Our standing assumptions are that $\mu$ is a countable limit ordinal, and Proposition 5.1 holds for all smaller ordinals. For the moment, assume also that there exists $\alpha<\mu$ such that $\Phi_{\xi}(\sigma)=\Phi_{\xi}(\tau)=\left\{x_{0}\right\}$ for all $\xi$ satisfying $\alpha<\xi<\mu$. The starting point for the induction step in this case is the following fact.

(17) There is an open and closed set $V$ in $\omega^{\mu}$ such that

(a) $V \cap\left(\omega^{\mu}\right)^{(\alpha+1)}=\varnothing$ and $V \cap\left(\omega^{\mu}\right)^{(\alpha)}$ is cofinal in $\omega^{\mu}$,

(b) $\Phi_{\xi}(\sigma \mid V)=\Phi_{\xi}(\tau \mid V)=\Phi_{\xi}(\sigma)$ for all $\xi \leqq \alpha$,

(c) $\lim _{\eta \rightarrow \omega^{\mu}, \eta \in \omega^{\mu}-v} d(\sigma(\eta), \tau(\eta))=0$, and

(d) $d(\sigma(\eta), \tau(\eta))<2 \delta$ for all $\eta \in \omega^{\mu}-V$.

Proof. By 5.5, there exist strictly increasing, sequences $\left\{\eta_{n}^{\prime} \mid n<\omega\right\}$ and $\left\{\eta_{n}^{\prime \prime} \mid n<\omega\right\}$ which are cofinal in $\omega^{\mu}$, contained in $\left(\omega^{\mu}\right)^{(\alpha)}-\left(\omega^{\mu}\right)^{(\alpha+1)}$, and satisfy $\Phi_{\alpha}(\sigma)=$ $\bigcap_{m<\omega}\left(\sigma\left(\left\{\eta_{n}^{\prime} \mid n \geqq m\right\}\right)\right)^{-}$and $\Phi_{\alpha}(\tau)=\bigcap_{m<\omega}\left(\tau\left(\left\{\eta_{n}^{\prime \prime} \mid n \geqq m\right\}\right)\right)^{-}$. Since $\Phi_{\alpha}(\sigma)=\Phi_{\alpha}(\tau)$, it is easy to see that the strictly increasing sequence $\left\{\eta_{n} \mid n<\omega\right\}$ obtained by arranging the set union $\left\{\eta_{n}^{\prime} \mid n<\omega\right\} \cup\left\{\eta_{n}^{\prime \prime} \mid n<\omega\right\}$ in ascending order also satisfies

(i) $\Phi_{\alpha}(\sigma)=\bigcap_{m<\omega}\left(\sigma\left(\left\{\eta_{n} \mid n \geqq m\right\}\right)\right)^{-}$, and

(ii) $\Phi_{\alpha}(\tau)=\bigcap_{m<\omega}\left(\tau\left(\left\{\eta_{n} \mid n \geqq m\right\}\right)\right)^{-}$. 
(The proof of this statement is essentially the same as the last step in the proof of 5.5.) Let

$$
\varepsilon=\min \{\delta-r(\sigma), \delta-r(\tau)\}
$$

Choose a decreasing sequence $\left\{\varepsilon_{n} \mid n<\omega\right\}$ of positive real numbers with $\varepsilon_{0} \leqq \varepsilon$, and $\lim _{n \rightarrow \omega} \varepsilon_{n}=0$. If $\rho \in\left(\omega^{\mu}\right)^{(\alpha+1)}$, then there is a unique $n<\omega$ such that $\eta_{n-1}<\rho<\eta_{n}$ (or $\rho<\eta_{0}$ if $n=0$ ). Choose $\zeta_{\rho}$ such that

(iii) $\eta_{n-1} \leqq \zeta_{\rho}<\rho$, and

(iv) $\zeta_{\rho} \leqq \eta \leqq \rho$ implies $d(\sigma(\eta), \sigma(\rho))<\varepsilon_{n}, d(\tau(\eta), \tau(\rho))<\varepsilon_{n}$.

It is possible to select such a $\zeta_{\rho}$ by the continuity of $\sigma$ and $\tau$. Define

$$
U=\bigcup_{\rho \in\left(\omega^{\mu}\right)^{(\alpha+1)}}\left(\zeta_{\rho}, \rho\right], \quad V=\omega^{\mu}-U
$$

By 5.3, $U$ and $V$ are open and closed, and $U \supseteq\left(\omega^{\mu}\right)^{(\alpha+1)}$, so that $V \cap\left(\omega^{\mu}\right)^{(\alpha+1)}=\varnothing$. On the other hand, by (iii), $V \supseteq\left\{\eta_{n} \mid n<\omega\right\}$, so that $V \cap\left(\omega^{\mu}\right)^{(\alpha)}$ is cofinal in $\omega^{\mu}$. Suppose that $\eta \in U$. Then there exists $\rho \in\left(\omega^{\mu}\right)^{(\alpha+1)}$, and $n<\omega$ such that $\eta_{n-1} \leqq \zeta_{\rho}$ $<\eta \leqq \rho<\eta_{n}$.

Consequently,

$$
\begin{aligned}
d(\sigma(\eta), \tau(\eta)) & \leqq d(\sigma(\eta), \sigma(\rho))+d\left(\sigma(\rho), x_{0}\right)+d\left(\tau(\rho), x_{0}\right)+d(\tau(\eta), \tau(\rho)) \\
& <d\left(\sigma(\rho), x_{0}\right)+d\left(\tau(\rho), x_{0}\right)+2 \varepsilon_{n} .
\end{aligned}
$$

Since $\rho \in\left(\omega^{\mu}\right)^{(\alpha+1)}$ and $\Phi_{\alpha+1}(\sigma)=\Phi_{\alpha+1}(\tau)=\left\{x_{0}\right\}$, it follows that

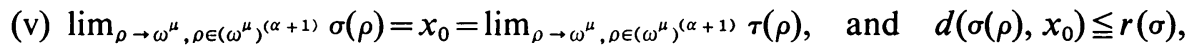
$d\left(\tau(\rho), x_{0}\right) \leqq r(\tau)$ for all $\rho \in\left(\omega^{\mu}\right)^{(\alpha+1)}$. Thus, $\lim _{\eta \rightarrow \omega^{\mu}, \eta \in V} d(\sigma(\eta), \tau(\eta))=0$ and $d(\sigma(\eta), \tau(\eta))<r(\sigma)+r(\tau)+(\delta-r(\sigma))+(\delta-r(\tau))=2 \delta$. It is an obvious consequence of (i) and (ii) that $\Phi_{\alpha}(\sigma \mid V) \supseteq \Phi_{\alpha}(\sigma)$ and $\Phi_{\alpha}(\tau \mid V) \supseteq \Phi_{\alpha}(\tau)$. The reverse inclusions are clear from 4.2, so that $\Phi_{\alpha}(\sigma \mid V)=\Phi_{\alpha}(\tau \mid V)=\Phi_{\alpha}(\sigma)$. If $\xi<\alpha$, then $x_{0} \in \Phi_{\alpha}(\sigma)=$ $\Phi_{\alpha}(\sigma \mid V) \subseteq \Phi_{\xi}(\sigma \mid V)$. Similarly, $x_{0} \in \Phi_{\xi}(\tau \mid V)$. By (iv) and (v), $\lim _{\eta \rightarrow \omega^{\mu}, \eta \in U} \sigma(\eta)=x_{0}$ and $\lim _{\eta \rightarrow \omega^{\mu}, \eta \in U} \tau(\eta)=x_{0}$. Hence, $\Phi_{\xi}(\sigma \mid U)=\Phi_{\xi}(\tau \mid U)=\left\{x_{0}\right\}$ for all $\xi<\mu$. Therefore, by $4.2, \quad \Phi_{\xi}(\sigma)=\Phi_{\xi}(\sigma \mid U) \cup \Phi_{\xi}(\sigma \mid V)=\left\{x_{0}\right\} \cup \Phi_{\xi}(\sigma \mid V)=\Phi_{\xi}(\sigma \mid V)$, and similarly, $\Phi_{\xi}(\tau)=\Phi_{\xi}(\tau \mid V)$ for all $\xi<\alpha$. This completes the proof of the statement (17).

The induction hypothesis can now be used to carry out the induction step in our special case $\Phi_{\xi}(\sigma)=\Phi_{\xi}(\tau)=\left\{x_{0}\right\}$ for $\alpha<\xi<\mu$. Let $U$ and $V$ be as in (17). By 5.4, $V \sim \omega^{\alpha+1}$. From (17b), we have $r(\sigma, V) \leqq r(\sigma)<\delta$ and $r(\tau, V) \leqq r(\tau)<\delta$. Thus, by (17b) again, the induction hypothesis is applicable: there exists a homeomorphism $\chi_{0}$ of $V$ onto itself such that

and

$$
\lim _{\eta \rightarrow \omega^{\mu}, \eta \in V} d\left(\sigma(\eta), \tau\left(\chi_{0}(\eta)\right)\right)=0
$$

$$
d\left(\sigma(\eta), \tau\left(\chi_{0}(\eta)\right)\right)<2 \delta \text { for all } \eta \in V .
$$

Define $\chi: \omega^{\mu} \rightarrow \omega^{\mu}$ by $\chi(\eta)=\chi_{0}(\eta)$ for $\eta \in V, \chi(\eta)=\eta$ for $\eta \in U$. Clearly $\chi$ is a homeomorphism of $\omega^{\mu}$ onto itself. By (17c), (17b) and the properties of $\chi_{0}$ listed above, it is clear that $\chi$ satisfies 5.1(a) and (b). 
We will now show that the general case of the induction step can be deduced from the special case that has just been treated, using Lemma 5.2. All notation will be as in 5.2. By 5.2(e) and 5.4, each of the sets $U_{n}$ and $V_{n}$ is order isomorphic to $\omega^{\mu}$. Thus, all of the hypotheses of the special case are satisfied when $U_{n}$ and $V_{n}$ are identified with $\omega^{\mu}$. Consequently, for each $n<\omega$, there is a homeomorphism $\chi_{n}$ of $U_{n}$ to $V_{n}$ such that

and

$$
\lim _{\eta \rightarrow \omega^{\mu}, \eta \in U_{n}} d\left(\sigma(\eta), \tau\left(\chi_{n}\right)\right)=0,
$$

$$
d\left(\sigma(\eta), \tau\left(\chi_{n}(\eta)\right)\right)<2 \delta_{n} \text { for all } \eta \in U_{n} .
$$

Define $\chi=\bigcup_{n<\omega} \chi_{n}$. Plainly, $\chi$ is a one-to-one mapping of $\omega^{\mu}$ onto $\omega^{\mu}$ such that $d(\sigma(\eta), \tau(\chi(\eta)))<2 \delta$ for all $\eta \in \omega^{\mu}$. Let $\varepsilon>0$. Since $\lim _{n \rightarrow \omega} \delta_{n}=0$, there exists $m<\omega$ such that $\delta_{n}<\varepsilon / 2$ for all $n \geqq m$. Consequently, $\eta \in U_{n}, n \geqq m$ implies $d(\sigma(\eta), \tau(\chi(\eta)))$ $<\varepsilon$. Since

$$
\lim _{\eta \rightarrow \omega^{\mu}, \eta \in U_{n}} d\left(\sigma(\eta), \tau\left(\chi_{n}(\eta)\right)\right)=0 \text { for all } n,
$$

there exists $\zeta<\omega^{\mu}$ such that if $\eta \geqq \zeta$ and $\eta \in \bigcup_{n<m} U_{n}$, then $d(\sigma(\eta), \tau(\chi(\eta)))<\varepsilon$. Therefore, by the choice of $m$, this inequality holds for every $\eta \geqq \zeta$. This argument proves that $\lim _{\eta \rightarrow \omega^{\mu}} d(\sigma(\eta), \tau(\chi(\eta)))=0$. It remains to show that $\chi$ is a homeomorphism. Let $W \subseteq \omega^{\mu}$ be open. Then $W \cap V_{n}$ is open for each $n$. Consequently, $\chi^{-1}\left(W \cap V_{n}\right)=\chi_{n}^{-1}\left(W \cap V_{n}\right)$ is open. Finally, $\chi^{-1}(W)=\bigcup_{n<\omega} \chi^{-1}\left(W \cap V_{n}\right)$ is open in $\omega^{\mu}$. This shows that $\chi$ is continuous. Similarly, $\chi^{-1}$ is continuous. Thus, $\chi$ is a homeomorphism.

The final job is to prove Lemma 5.2. We will approach this result through a sequence of technical lemmas.

Lemma 5.6. Let $\sigma: \omega^{\mu} \rightarrow W$ be continuous. Then $\lim _{\xi \rightarrow \mu} r_{\xi}(\sigma)=0$.

Proof. Denote

$$
T=\bigcap_{\xi<\mu} \Phi_{\xi}(\sigma)=\bigcap_{\eta<\omega^{\mu}} \bigcap_{\xi<\mu}\left(\sigma\left(\left(\omega^{\mu}\right)^{(\xi)} \cap\left[\eta, \omega^{\mu}\right)\right)\right)^{-} .
$$

Let $\varepsilon>0$ be arbitrary. Using various properties of $e$ which were listed earlier, we conclude

(i) there exists $\eta_{0}<\omega^{\mu}$ such that

$$
e\left(\bigcap_{\xi<\mu}\left(\sigma\left(\left(\omega^{\mu}\right)^{(\xi)} \cap\left[\eta_{0}, \omega^{\mu}\right)\right)\right)^{-}, T\right)<\varepsilon / 2,
$$

(ii) there exists $\xi_{0}<\mu$ such that $\omega^{\xi_{0}}>\eta_{0}$ and for all $\xi \geqq \xi_{0}$,

$$
e\left(\left(\sigma\left(\left(\omega^{\mu}\right)^{(\xi)} \cap\left[\eta_{0}, \omega^{\mu}\right)\right)\right)^{-}, \bigcap_{\xi<\mu}\left(\sigma\left(\left(\omega^{\mu}\right)^{(\xi)} \cap\left[\eta_{0}, \omega^{\mu}\right)\right)\right)^{-}\right)<\varepsilon / 2 .
$$

By (i) and (ii), it follows that

(iii) $\xi \geqq \xi_{0}$ implies $e\left(\left(\sigma\left(\left(\omega^{\mu}\right)^{(\xi)} \cap\left[\eta_{0}, \omega^{\mu}\right)\right)\right)^{-}, T\right)<\varepsilon$. Clearly, if $\xi \geqq \xi_{0}$, then $\omega^{\xi} \geqq \omega^{\xi}>\eta_{0}$ so that $\left(\omega^{\mu}\right)^{(\xi)} \subseteq\left[\eta_{0}, \omega^{\mu}\right)$. Therefore, $\sigma\left(\left(\omega^{\mu}\right)^{(\xi)} \cap\left[\eta_{0}, \omega^{\mu}\right)\right)=\sigma\left(\left(\omega^{\mu}\right)^{(\xi)}\right)$. 
Finally, note that $\left(\sigma\left(\left(\omega^{\mu}\right)^{(\xi)}\right)\right)^{-} \supseteq \Phi_{\xi}(\sigma) \supseteq T$ implies

$$
r_{\xi}(\sigma)=e\left(\sigma\left(\left(\omega^{\mu}\right)^{(\xi)}\right), \Phi_{\xi}(\sigma)\right)=e\left(\left(\sigma\left(\omega^{\mu}\right)^{(\xi)}\right)^{-}, \Phi_{\xi}(\sigma)\right) \leqq e\left(\left(\sigma\left(\left(\omega^{\mu}\right)^{(\xi)}\right)\right)^{-}, T\right)<\varepsilon
$$

for all $\xi \geqq \xi_{0}$. Since $\varepsilon>0$ was arbitrary, this completes the proof.

In the next four lemmas, the following hypotheses are assumed to be in effect:

$U$ is a full subset of $\omega^{\mu}$, that is, $U$ is open and closed, and $U \cap\left(\omega^{\mu}\right)^{(\xi)}$ is cofinal in $\omega^{\mu}$ for all $\xi<\mu$;

$\sigma$ is a continuous mapping of $\omega^{\mu}$ to $W$.

LeMmA 5.7. Let $\varepsilon>0$. Choose any $x_{0}$ in $\bigcap_{\xi<\mu} \Phi_{\xi}(\sigma \mid U)$. Then there is a set $V \subseteq U$ such that

(a) $V$ and $M=U-V$ are full in $\omega^{\mu}$,

(b) $r(\sigma, V)<\varepsilon$,

(c) $\Phi_{\xi}(\sigma \mid V)=\left\{x_{0}\right\}$ for all $\xi<\mu$,

(d) $\Phi_{\xi}(\sigma \mid M)=\Phi_{\xi}(\sigma \mid U)$ for all $\xi<\mu$.

Proof. Let $\left\{\alpha_{n} \mid n<\omega\right\}$ be an increasing sequence of nonlimit ordinals such that $\lim _{n \rightarrow \omega} \alpha_{n}=\mu$. Let $\left\{\varepsilon_{n} \mid n<\omega\right\}$ be a decreasing sequence of positive real numbers with $\varepsilon_{0}<\varepsilon / 3$, and $\lim _{n \rightarrow \omega} \varepsilon_{n}=0$. Choose a strictly increasing sequence $\left\{\eta_{n} \mid n<\omega\right\}$ such that $\eta_{n} \in U^{\left(\alpha_{n}\right)}, d\left(\sigma\left(\eta_{n}\right), x_{0}\right)<\varepsilon_{n}$.

For each $n<\omega$, choose $\zeta_{n} \in U^{\left(\alpha_{n}-1\right)}$ such that $\eta_{n-1}<\zeta_{n}<\eta_{n}\left(\zeta_{0}<\eta_{0}\right)$, and $\zeta_{n} \leqq \zeta \leqq \eta_{n}$ implies $d\left(\sigma(\zeta), \sigma\left(\eta_{n}\right)\right)<\varepsilon_{n}$. Let $V=\bigcup_{n<\omega}\left(\zeta_{n}, \eta_{n}\right]$. Then $V$ is full. Since $\zeta_{n} \in M=U-V$, it follows that $M$ is also full. By construction, $d\left(\sigma(\zeta), x_{0}\right)<(2 / 3) \varepsilon$ for all $\zeta \in V$. Therefore, $r(\sigma, V)<\varepsilon$. Also, it is evident that $\lim _{\eta \rightarrow \omega^{\mu}, \eta \in V} \sigma(\eta)=x_{0}$. Hence, $\Phi_{\xi}(\sigma \mid V)=\left\{x_{0}\right\}$ for all $\xi<\mu$. Since $\lim _{n \rightarrow \omega} \sigma\left(\zeta_{n}\right)=x_{0}$, we have $x_{0} \in \Phi_{\xi}(\sigma \mid M)$. By 4.2, $\Phi_{\xi}(\sigma \mid U)=\Phi_{\xi}(\sigma \mid V) \cup \Phi_{\xi}(\sigma \mid M)=\left\{x_{0}\right\} \cup \Phi_{\xi}(\sigma \mid M)=\Phi_{\xi}(\sigma \mid M)$ for all $\xi<\mu$.

LEMMA 5.8. Let $\delta$ be a positive real number, and $\alpha<\mu$ an ordinal number such that if $\alpha \leqq \xi<\mu$, then $e\left(\sigma\left(U \cap\left(\omega^{\mu}\right)^{(\xi)}\right), \Phi_{\xi}(\sigma \mid U)\right)<\delta$. Then there exists $V \subseteq U$ such that

(a) $V$ is full in $\omega^{\mu}$,

(b) $V \supseteq U \cap\left(\omega^{\mu}\right)^{(\alpha)}$,

(c) $r(\sigma, V)<2 \delta$,

(d) $\Phi_{\xi}(\sigma \mid V)=\Phi_{\alpha}(\sigma \mid U)$ for $\xi \leqq \alpha$,

(e) $\Phi_{\xi}(\sigma \mid V)=\Phi_{\xi}(\sigma \mid U)$ for $\xi \geqq \alpha$.

Proof. Since $e\left(\left(\sigma\left(U \cap\left(\omega^{\mu}\right)^{(\alpha)}\right)\right)^{-}, \Phi_{\alpha}(\sigma \mid U)\right)=e\left(\sigma\left(U \cap\left(\omega^{\mu}\right)^{(\alpha)}\right), \Phi_{\alpha}(\sigma \mid U)\right)<\delta$, it follows that

$$
\sigma\left(U \cap\left(\omega^{\mu}\right)^{(\alpha)}\right)^{-} \subseteq\left\{x \in W \mid d\left(x, \Phi_{\alpha}(\sigma \mid U)\right)<\delta\right\} .
$$

By compactness and the zero dimensionality of $W$, there is an open and closed set $N$ such that

$$
\sigma\left(U \cap\left(\omega^{\mu}\right)^{(\alpha)}\right) \subseteq N \subseteq\left\{x \in W \mid d\left(x, \Phi_{\alpha}(\sigma \mid U)\right)<\delta\right\} .
$$

Let $M=\sigma^{-1}(N) \cap U$. Then $M$ is open and closed, and $M \supseteq U \cap\left(\omega^{\mu}\right)^{(\alpha)}$. Let $\left\{\alpha_{n} \mid n<\omega\right\}$ be a strictly increasing sequence of ordinals such that $\lim _{n \rightarrow \omega} \alpha_{n}=\mu$. 
Choose a decreasing sequence $\left\{\varepsilon_{n} \mid n<\omega\right\}$ of real numbers with $\varepsilon_{0}<\delta$ and $\lim _{n \rightarrow \omega} \varepsilon_{n}$ $=0$. By the continuity of $\sigma$, there exist ordinal numbers $\zeta_{\eta}$ associated with each $\eta \in U \cap\left(\omega^{\mu}\right)^{(\alpha)}$ such that $\left(\zeta_{\eta}, \eta\right] \subseteq M$, if $\omega^{\alpha_{n}}<\eta<\omega^{\alpha_{n+1}}$, then $\zeta_{\eta} \geqq \omega^{\alpha_{n}}$, and $d(\sigma(\rho), \sigma(\eta))$ $<\varepsilon_{n}$ for all $\rho \in\left(\zeta_{\eta}, \eta\right]$.

Define $V=\bigcup_{\eta \in U \cap\left(\omega^{\mu}\right)^{(\alpha)}}\left(\zeta_{\eta}, \eta\right]$. By 5.3, $V$ is open and closed. Clearly, $U \supseteq V \supseteq U$ $\cap\left(\omega^{\mu}\right)^{(\alpha)}$, so that $V \cap\left(\omega^{\mu}\right)^{(\xi)}=U \cap\left(\omega^{\mu}\right)^{(\xi)}$ for all $\xi \geqq \alpha$. In particular, $V$ is full in $\omega^{\mu}$, (e) holds, and

$$
e\left(\sigma\left(V \cap\left(\omega^{\mu}\right)^{(\xi)}\right), \Phi_{\xi}(\sigma \mid V)\right) \leqq e\left(\sigma\left(U \cap\left(\omega^{\mu}\right)^{(\xi)}\right), \Phi_{\xi}(\sigma \mid U)\right)<\delta
$$

for $\xi \geqq \alpha$. If $\rho \in V \cap\left(\omega^{\alpha_{n}}, \omega^{\alpha_{n+1}}\right]$, then

$$
\begin{aligned}
d\left(\sigma(\rho), \Phi_{\alpha}(\sigma \mid U)\right) & \leqq \varepsilon_{n}+e\left(\sigma\left(U \cap\left(\omega^{\mu}\right)^{(\alpha)} \cap\left(\omega^{\alpha}, \omega^{\mu}\right)\right), \Phi_{\alpha}(\sigma \mid U)\right) \\
& \leqq \varepsilon_{n}+e\left(\sigma\left(U \cap\left(\omega^{\mu}\right)^{(\alpha)}\right), \Phi_{\alpha}(\sigma \mid U)\right) \\
& \leqq \varepsilon_{0}+\delta
\end{aligned}
$$

It follows that $\Phi_{\xi}(\sigma \mid V) \subseteq \Phi_{\alpha}(\sigma \mid U)=\Phi_{\alpha}(\sigma \mid V) \subseteq \Phi_{\xi}(\sigma \mid V)$ for all $\xi \leqq \alpha$, and $r(\sigma, V)$ $\leqq \varepsilon_{0}+\delta<2 \delta$.

Lemma 5.9. Let $\alpha<\mu$. Then there is an open and closed subset $V$ of $U$ such that

(a) $V \cap\left(\omega^{\mu}\right)^{(\alpha+1)}=\varnothing$,

(b) $V \cap\left(\omega^{\mu}\right)^{(\alpha)}$ is cofinal in $\omega^{\mu}$, and

(c) $\Phi_{\alpha}(\sigma \mid V)=\Phi_{\alpha}(\sigma \mid U)$.

Proof. Choose a decreasing sequence $\left\{\varepsilon_{n} \mid n<\omega\right\}$ of positive real numbers with $\varepsilon_{0}<r(\sigma, U)$, and $\lim _{n \rightarrow \omega} \varepsilon_{n}=0$. Let $\left\{\eta_{n} \mid n<\omega\right\}$ be a sequence of ordinals which satisfies the conditions of 5.5. Since $\eta_{n} \notin\left(\omega^{\mu}\right)^{(\alpha+1)}$, either $\eta_{n}$ is the least element of $\left(\omega^{\mu}\right)^{(\alpha)}$, or else $\eta_{n}$ has a predecessor $\zeta_{n}$ in $\left(\omega^{\mu}\right)^{(\alpha)}$. We can assume that the latter condition holds for all $n$. (Otherwise, delete $\eta_{0}$.) Define $V=\cup_{n<\omega}\left(\zeta_{n}, \eta_{n}\right]$. Since $\left\{\eta_{n} \mid n<\omega\right\}$ is cofinal in $\omega^{\mu}$, it is clear that $V$ is open and closed. Since $\left(\zeta_{n}, \eta_{n}\right]$ $\cap\left(\omega^{\mu}\right)^{(\alpha+1)}=\varnothing$ for all $n$, it follows that $V \cap\left(\omega^{\mu}\right)^{(\alpha+1)}=\varnothing$. Plainly, $V \cap\left(\omega^{\mu}\right)^{(\alpha)}$ $=\left\{\eta_{n} \mid n<\omega\right\}$ is cofinal in $\omega^{\mu}$, and

$$
\Phi_{\alpha}(\sigma \mid V)=\bigcap_{\eta<\omega^{\mu}}\left(\sigma\left(V \cap\left(\omega^{\mu}\right)^{(\alpha)} \cap\left(\eta, \omega^{\mu}\right)\right)\right)^{-}=\bigcap_{m<\omega}\left(\sigma\left(\left\{\eta_{n} \mid n \geqq m\right\}\right)\right)^{-}=\Phi_{\alpha}(\sigma \mid U)
$$

(by 5.5).

LEMMA 5.10. In addition to the standing hypotheses, assume that $x_{0} \in \bigcap_{\xi<\mu} \Phi_{\xi}(\sigma \mid U)$, $\delta$ is any positive real number, $\varepsilon$ is a positive real number which satisfies $\varepsilon \geqq r(\sigma, U)$, $\beta<\mu$, and $\alpha \in(\beta, \mu)$ is such that $r_{\xi}(\sigma, U)<\delta$ for all $\xi \in(\alpha, \mu)$. Then there exists $V \subseteq U$ such that

(a) $V$ and $M=U-V$ are full subsets of $\omega^{\mu}$,

(b) $V \supseteq U \cap\left[0, \omega^{\beta}\right]$,

(c) $r(\sigma, V) \leqq \varepsilon, r(\sigma, M)<2 \delta$,

(d) $\Phi_{\xi}(\sigma \mid V)=\Phi_{\xi}(\sigma \mid U)$ for $\xi \leqq \alpha, \Phi_{\xi}(\sigma \mid V)=\left\{x_{0}\right\}$ for $\xi>\alpha$, and

(e) $\Phi_{\xi}(\sigma \mid M)=\Phi_{\xi}(\sigma \mid U)$ for $\xi>\alpha, \Phi_{\xi}(\sigma \mid M)=\Phi_{\alpha+1}(\sigma \mid U)$ for $\xi<\alpha$. 
Proof. By 5.8, there exists $U_{1} \subseteq U$ such that $U_{1}$ is full in $\omega^{\mu}$

and

$$
\begin{array}{ll}
U_{1} \supseteq U \cap\left(\omega^{\mu}\right)^{(\alpha+1)}, & r\left(\sigma, U_{1}\right)<2 \delta \\
\Phi_{\xi}\left(\sigma \mid U_{1}\right)=\Phi_{\alpha+1}(\sigma \mid U) & \text { for } \xi \leqq \alpha+1,
\end{array}
$$

$$
\Phi_{\xi}\left(\sigma \mid U_{1}\right)=\Phi_{\xi}(\sigma \mid U) \quad \text { for } \xi \geqq \alpha+1 \text {. }
$$

By 5.7, there exists $V_{1} \subseteq U_{1}$ such that $V_{1}$ and $U_{2}=U_{1}-V_{1}$ are full in $\omega^{\mu}$ and satisfy

$$
\begin{aligned}
& r\left(\sigma, V_{1}\right)<\varepsilon, \\
& \Phi_{\xi}\left(\sigma \mid V_{1}\right)=\left\{x_{0}\right\} \quad \text { for all } \xi<\mu, \text { and }
\end{aligned}
$$

(i) $\Phi_{\xi}\left(\sigma \mid U_{2}\right)=\Phi_{\xi}\left(\sigma \mid U_{1}\right)$ for all $\xi<\mu$.

By 5.9, there exists $V_{2} \subseteq U_{2}$ such that $V_{2}$ is open and closed in $\omega^{\mu}$,

(ii) $V_{2} \cap\left(\omega^{\mu}\right)^{(\alpha+1)}=\varnothing$ and $V_{2} \cap\left(\omega^{\mu}\right)^{(\alpha)}$ is cofinal in $\omega^{\mu}$, and

(iii) $\Phi_{\alpha}\left(\sigma \mid V_{2}\right)=\Phi_{\alpha}\left(\sigma \mid U_{2}\right)$.

Define

Note that

$$
M=\left(U_{2}-V_{2}\right) \cap\left(\omega^{\beta}, \omega^{\mu}\right), \quad V=U-M
$$

(iv) $V=\left(U-V_{1}\right) \cup V_{1} \cup V_{2} \cup\left(U \cap\left[0, \omega^{\beta}\right]\right)$.

Clearly, $V$ and $M$ are open and closed, and since $V_{1}$ is full, so is $V$. It follows from (ii) and the fact that $U_{2}$ is full that $U_{2}-V_{1}$, and hence also $M$, is full. Using (i), (ii), and 4.2, we obtain $\Phi_{\xi}(\sigma \mid M)=\Phi_{\xi}\left(\sigma \mid\left(U_{2}-V_{2}\right)\right)=\Phi_{\xi}\left(\sigma \mid U_{2}\right)=\Phi_{\xi}\left(\sigma \mid U_{1}\right)=\Phi_{\xi}(\sigma \mid U)$ for all $\xi \geqq \alpha+1$. If $\xi \leqq \alpha$, then

$$
\begin{aligned}
\Phi_{\alpha+1}(\sigma \mid U) & =\Phi_{\alpha+1}\left(\sigma \mid\left(U_{2}-V_{2}\right)\right) \subseteq \Phi_{\xi}\left(\sigma \mid\left(U_{2}-V_{2}\right)\right) \subseteq \Phi_{\xi}\left(\sigma \mid U_{2}\right) \subseteq \Phi_{\xi}\left(\sigma \mid U_{1}\right) \\
& =\Phi_{\alpha+1}(\sigma \mid U) .
\end{aligned}
$$

This proves (e) and shows that $\Phi_{\xi}(\sigma \mid M)=\Phi_{\xi}\left(\sigma \mid U_{1}\right)$ for all $\xi<\mu$. Therefore, since $M \subseteq U_{2} \subseteq U_{1}$, it follows that $r(\sigma, M) \leqq r\left(\sigma, U_{1}\right)<2 \delta$. We next compute $\Phi_{\xi}(\sigma \mid V)$. Suppose first that $\xi \geqq \alpha+1$. Then by (ii)

(v) $V \cap\left(\omega^{\mu}\right)^{(\xi)}=V_{1} \cap\left(\omega^{\mu}\right)^{(\xi)}$.

Consequently, $\Phi_{\xi}(\sigma \mid V)=\Phi_{\xi}\left(\sigma \mid V_{1}\right)=\left\{x_{0}\right\}$ for $\xi \geqq \alpha+1$. If $\xi \leqq \alpha$, then by (i) and (iii) we obtain

$$
\Phi_{\xi}(\sigma \mid V) \supseteq \Phi_{\alpha}(\sigma \mid V) \supseteq \Phi_{\alpha}\left(\sigma \mid V_{2}\right)=\Phi_{\alpha}\left(\sigma \mid U_{2}\right)=\Phi_{\alpha}\left(\sigma \mid U_{1}\right)=\Phi_{\alpha+1}(\sigma \mid U) .
$$

Hence, by 4.2 ,

$$
\Phi_{\xi}(\sigma \mid U)=\Phi_{\xi}(\sigma \mid V) \cup \Phi_{\xi}(\sigma \mid M)=\Phi_{\xi}(\sigma \mid V) \cup \Phi_{\alpha+1}(\sigma \mid U)=\Phi_{\xi}(\sigma \mid V) .
$$

This finishes the proof of (d). If $\xi \geqq \alpha+1$, then by (v) we have $r_{\xi}(\sigma, V)=r_{\xi}\left(\sigma, V_{1}\right)$ $\leqq r\left(\sigma, V_{1}\right)<\varepsilon$. On the other hand, for $\xi \leqq \alpha, \Phi_{\xi}(\sigma \mid V)=\Phi_{\xi}(\sigma \mid U)$ and $V \subseteq U$, so that $r_{\xi}(\sigma, V) \leqq r_{\xi}(\sigma, U) \leqq r(\sigma, U) \leqq \varepsilon$. Consequently, $r(\sigma, V) \leqq \varepsilon$.

Proof of Lemma 5.2. We construct the $\alpha_{n}$, $U_{n}$, and $V_{n}$ recursively. Assume that $\alpha_{j}, U_{j}$, and $V_{j}$ have been chosen for $j<n$ satisfying 
(i) $\beta_{j}<\alpha_{j}<\mu$,

(ii) each $U_{j}$ and $V_{j}$ is full in $\omega^{\mu}$,

(iii) $\bigcup_{j<n} U_{j} \supseteq\left[0, \omega^{\beta_{n-1}}\right], \bigcup_{j<n} V_{j} \supseteq\left[0, \omega^{\beta_{n-1}}\right]$,

(iv) $U_{i} \cap U_{j}=V_{i} \cap V_{j}=\varnothing$ if $i \neq j$,

(v) $r\left(\sigma, U_{j}\right)<\delta_{j}, r\left(\tau, V_{j}\right)<\delta_{j}$ for all $j<n$,

(vi) $\Phi_{\xi}\left(\sigma \mid U_{j}\right)=\Phi_{\xi}\left(\tau \mid V_{j}\right)=\left\{x_{0}\right\}$ if $\xi>\alpha_{j}$,

(vii) $\Phi_{\xi}\left(\sigma \mid U_{j}\right)=\Phi_{\xi}\left(\tau \mid V_{j}\right)=\Phi_{\xi}(\sigma)$ if $j>0$ and $\alpha_{j-1}<\xi \leqq \alpha_{j}$, or $j=0$ and $\xi \leqq \alpha_{0}$,

(viii) $\Phi_{\xi}\left(\sigma \mid U_{j}\right)=\Phi_{\xi}\left(\tau \mid V_{j}\right)=\Phi_{\alpha_{j-1}+1}(\sigma)$ if $j>0$ and $\xi \leqq \alpha_{j-1}$.

If $X_{n}=\omega^{\mu} \bigcup_{j<n} U_{n}, Y_{n}=\omega^{\mu}-\bigcup_{j<n} V_{j}$, then

(ix) $\Phi_{\xi}\left(\sigma \mid X_{n}\right)=\Phi_{\xi}\left(\tau \mid Y_{n}\right)=\Phi_{\xi}(\sigma)$ for $\xi>\alpha_{n-1}$,

(x) $\Phi_{\xi}\left(\sigma \mid X_{n}\right)=\Phi_{\xi}\left(\tau \mid Y_{n}\right)=\Phi_{\alpha_{n-1}+1}(\sigma)$ for $\xi \leqq \alpha_{n-1}$, and

(xi) $r\left(\sigma, X_{n}\right)<\delta_{n}, r\left(\tau, Y_{n}\right)<\delta_{n}$.

If $n=0$, these conditions are satisfied vacuously, except for the last two, which are valid by hypothesis. By 5.6, it is possible to choose $\alpha_{n}<\mu$ such that $\alpha_{n}>\beta_{n}, \alpha_{n}>\alpha_{n-1}$, and $e\left(\sigma\left(X_{n} \cap\left(\omega^{\mu}\right)^{(\xi)}\right), \Phi_{\xi}\left(\sigma \mid X_{n}\right)\right)<(1 / 2) \delta_{n+1}, e\left(\tau\left(Y_{n} \cap\left(\omega^{\mu}\right)^{(\xi)}\right), \Phi_{\xi}\left(\tau \mid Y_{n}\right)\right)<(1 / 2) \delta_{n+1}$ for all $\xi$ satisfying $\alpha_{n}<\xi<\mu$. The desired $U_{n} \subseteq X_{n}, V_{n} \subseteq Y_{n}$ are now obtained by applying 5.10 to $X_{n}$ and $\sigma$, and to $Y_{n}$ and $\tau$ (with $\alpha=\alpha_{n}, \beta=\beta_{n}, \delta=(1 / 2) \delta_{n+1}$, and $\varepsilon=\delta_{n}$ ). The conditions (i)-(xi) are easily verified. It is clear that $\left\{\alpha_{n} \mid n<\omega\right\}$, $\left\{U_{n} \mid n<\omega\right\},\left\{V_{n} \mid n<\omega\right\}$ satisfy all of the conditions of 5.2.

This completes the proof of 5.2, so that we are done.

\section{REFERENCES}

1. P. R. Halmos, Lectures on Boolean algebras, Van Nostrand Math. Studies, no. 1, Van Nostrand, Princeton, N. J., 1963. MR 29 \#4713.

2. F. Hausdorff, Mengenlehre, Leipzig, 1927.

3. R. D. Mayer and R. S. Pierce, Boolean algebras with ordered bases, Pacific J. Math. 10 (1960), 925-942. MR 24 \#A696.

4. S. Mazurkiewicz and W. Sierpinski, Contribution à la topologie des ensembles dénombrables, Fund. Math. 1 (1920), 17-27.

5. R. S. Pierce, Introduction to the theory of abstract algebras, Holt, New York, 1968. MR 37 \#2655.

UNIVERSITY OF WASHINGTON, SEATtLe, WAShington 